\title{
Chinesisch als Rechtssprache im Kontext des Common Law: Der Fall Hongkong
}

\author{
Von Volker Pasternak
}

\section{Einleitung 1}

Am 30.6.1997 verliert Großbritannien seine letzte wichtige Kolonie, Hongkong, an die Volksrepublik China. Die Volksrepublik China hat sich für die Zeit nach dem Machtwechsel gegenüber Großbritannien völkerrechtlich verpflichtet, 2 neben dem kapitalistischen Wirtschaftssystem auch das Common Law3 in Hongkong für weitere 50 Jahre im wesentlichen unangetastet zu lassen. Trotzdem wird in Hongkong dem Souveränitätsübergang mit großer Sorge entgegengesehen. Groß ist das Mißtrauen gegen die übermächtigen kommunistischen Brüder aus dem Norden. Besonders seit den blutigen Ereignissen am 4.6.1989 in Peking formieren sich verschiedene politische Kräfte in und um Hongkong, um Hongkong für den Souveränitätswechsel zu wappnen, vorzubereiten, bzw. gefügig zu machen. Für eine angemessene Analyse des Chinesischen als Rechtssprache in Hongkong wird man sich deshalb in einem brisanten Spannungsfeld zwischen Sprache, Recht und Politik bewegen müssen. 4

1 Für die Transkription des Chinesischen wird hier die in der VR China gebräuchliche "Hanyu pinyin"-Umschrift verwendet, für das Kantonesische die eher akademische "Yale"-Umschrift und für das Japanische die "Hepbum"-Umschrift, soweit sich nicht für bestimmte Namen schon andere Transliterationen eingebürgert haben, was besonders beim Kantonesischen häufig vorkommt. Hervorhebungen in Zitaten stammen vom Verfasser. Die Arbeit reicht bis zum 1. März 1993.

2 Siehe dazu: "A Draft Agreement between the Govemment of the United Kingdom of Great Britain and Northem Ireland and the Govemment of the People's Republic of China on the Future of Hong Kong" (im folgenden kurz "Gemeinsame Erklärung" genannt), White Paper, London 26.9.1984; Deutsche Übersetzung in China aktuell, Heft 9/1984, S. 533 ff. Näheres, u.a. über die umstrittene Völkerrechtsqualität der Gerneinsamen Erklärung, im 3. Kapitel.

3 Hier: Common Law i.w.S. = Synonym für das anglo-amerikanische Recht im Gegensatz zum kontinentaleuropäischen Recht (Civil Law). Weitere Bedeutungen: 1. Gemeines Recht, das von den reisenden Richtem des königlichen Gerichts zu Westminster gebildet wurde; dem Billigkeitsrecht (equity) als Teil des Fallrechtes (case law) gegenübergestellt = Common Law i.e.S. 2. Synonym für das gesamte Fallrecht (case law); dem Gesetzesrecht (statute law) gegenübergestellt. - Terminologie nach $D$. Blwmenwitz, Einführung in das anglo-amerikanische Recht, JuS Schriftenreihe, 3. Aufl. München 1987, S. 4-5.

4 Eine ausf ührliche Analyse bleibt einer späteren Dissertation des Autors am Ostasiatischen Seminar der Universität Göttingen vorbehalten. Für Kritik und Anregungen jeder Art ist der Autor dankbar. 
Als Basiswissen seien zunächst einige allgemeinere Bemerkungen zur Sprachensituation in Hongkong vorweggeschickt: Die überwältigende Mehrheit der Bewohner5 der britischen Kronkolonie Hongkong6 sind ethnische Chinesen. Ihre Muttersprache ist Chinesisch7, meist in Form der kantonesischen Regionalsprache8. Abgesehen von Unterschieden im Stil ${ }^{9}$ gleicht die besonders im of fiziellen Zusammenhang als adäquat angesehene, gehobene Hongkonger Schriftsprache im Wortschatz weitgehend der im Mandarin 10-Sprachraum gebräuchlichen Schriftsprache, während geschriebenes Kantonesisch11 mit seiner stark idiomatischen Ausdruckskraft unter Verwendung von lokalen Schriftzeichen12 im vertraulichpersönlichen Bereich Anwendung findet.13 Viele Hongkonger empfinden die gehobene chinesische Schriftsprache aufgrund der Diskrepanz zum gesprochenen Alltagskantonesisch als fremd, wenn auch nicht in dem Maße wie das Englische. 14

5 Die Volkszählung vom 15.3.1991 ergab eine Einwohnerzahl von 5,67 Millionen. Unter Hinzurechnung der illegalen Einwanderer dürfte sich die tatsächliche Zahl der Einwohner allerdings auf über 6 Millionen belaufen. Siehe: Census and Statistics Department, Hong Kong 1991 Population Census, Summary Results, Govemment Printer, Hongkong 1991, Table 1, p. 32.

6 Offiziell wird aus politischen Erwägungen die Bezeichnung "Territory" bevoraugt.

7 Die Volkszählung vom 15.3.1991 (vgl. Fn. 5) gibe folgende Relationen bezüglich der im häuslichen Umfeld von den Hongkongem (Mindestalter: 5 Jahre) verwendeten Erstsprachen an: 88,7\% Kantonesisch; 7,1\% andere chinesische Sprachen (davon nur 1,1\% Mandarin(!), 1,4\% Chiu Chau, 1,6\% Hakka, 1,9\% Fukien (incl. Taiwanesisch), 0,4\% Sze Yap, 0,7 \% Shanghainesisch); 2,2 \% Englisch; siehe a.a.O., Table 11, p. 43.

Als weitere Sprache (z.B.: Zweit- oder Drittsprache) wird Kantonesisch von 7,1\%, Englisch von 29,4\% und Mandarin von nur $17 \%$ (!) der obigen Untersuchungsgruppe gesprochen; siehe a.a.O., Table 12, p. 43.

8 Der Autor hält die Bezeichnung "Dialekt" für ungeeignet, da dieser Begriff die eigenständige Bedeutung des Kantonesischen zu wenig zum Ausdruck bringt.

9 Eine nähere Analyse folgt im 6. Kapitel.

10 Chin.: Guanhua. Ursprünglich: Bezeichnung der qingzeitlichen Beamtensprache. Jetz: Hongkonger Bezeichnung für die nordchinesische Sprachgruppe; entspricht weitgehend den Offizialsprachen der Volksrepublik China (Pusonghua) und der Republik China auf Taiwan (Guoyu).

11 Siehe dazu R.S. Bauer, "Written Cantonese of Hong Kong", Cahiers de Linguistique Asie Orientale, Vol. XVII No. 2, Décembre 1988, pp. 245-293.

12 Lokale Schriftzeichen sind den Mandarin-Sprechem aus China und Taiwan nur selten verständlich. Die Jugendkultur in allen Teilen der chinesischen Welt scheint dem geschriebenen Kantonesisch gegenüber aufgeschlossener zu sein (Popmusik, Komikhefte, Filme).

13 Als Beispiele seien das "City Magazine", die Witzseiten in den Hongkonger Tageszeitungen und das Genre der "ghost stories" genannt.

14 Es wird kritisien, da $B$ sich Hongkonger Studenten schriftlich fehlerf rei und tiefsinnig weder im Englischen noch im Chinesischen auszudrücken vermöchten. Während dieses Defizit für die wirtschaftliche Entwicklung Hongkongs irrelevant sei, wirke es sich im kulturellen Bereich deutlich aus. Dazu: Luke Kang Kwang (ed.), Issues of Language and Education, Linguistic Society of Hong Kong, Hongkong 1992. 



Rechtskonzepte aus dem europäischen Kulturraum stammen. Vomehmlich über das Studium der englischen Sprache, die Rechtsvokabular verschiedener indo-europäischer Sprachen absorbiert hat ${ }^{16}$, gewannen die Hongkonger bisher einen vom sprachlichen Bildungsgr ad abhängigen Eindruck von den Rechtskonzepten. Im Zuge der Vorbereitungen in Hongkong auf den britisch-chinesischen Souveränitätsübergang17 am 1.7.1997 gewinnt Chinesisch als Rechtssprache zunehmend an Bedeutung. Daraus ergeben sich folgende Fragen:

- Wie bedeutend ist das Kommunikationsmedium englische Sprache für das Verständnis des Common Law?

- Kann es ein chinesisches Common Law geben?

Ganz allgemein ist in der Rechtsvergleichung die Problematik der verlustfreien Ubersetzbarkeit von Rechtstexten umstritten. 18 VerläBt man nun den indoeuropäischen Sprachraum, in dem sich diese Untersuchungen bewegen, und wendet sich der Ubersetzbarkeit des englischen Common Law in das Chinesische zu, so ergeben sich neue Aspekte, die in der kulturellen Tradition und der ideographischen Schrift der Chinesen ihre Wurzeln haben. In tief erschürfenden Beurteilungen der Uberlebenschance eines chinesischen Common Law in Hongkong haben einige Experten bereits Stellung zu diesen Fragen bezogen. 19

Nachdem im folgenden die historische Entwicklung in Hongkong bezüglich des Chinesischen als Rechtssprache nachgezeichnet und eine auf den rechtlichen und tatsächlichen Rahmenbedingungen beruhende Prognose für die Zeit nach 1997 angestellt wird, sollen die in Hongkong sich zunehmend stabilisierende chinesische Common Law-Terminologie und der Stil der Rechtssprache einer rechtsvergleichenden Analyse unterzogen werden. Dabei

15 Wichtigste Rechtsquellen des Common Law i.w.S. sind das Common Law i.e.S., die rules of equity und das statute law, das in Hongkong aus ordinances und subsidiary legislation besteht. Derzeit finden noch einige Acts of Parliament (G.B.) in Hongkong Anwendung, die aber bis 1997 durch lokale Gesetzgebung ersetzt werden sollen.

16 Instruktiv au dieser Frage einerseits: D. Roebuck, "The Chronology of a Cobweb: Anglo-Norman from 1066 to 1250"; Working Papers in Languages and Linguistics; (1990) 2; City Polytechnic of Hong Kong; pp. 1-43, bezüglich des "law French"; andererseits: N. Bird, A First Handbook of the Roots of English, Jersey/Hongkong 1990, bezüglich indo-europäischer "word roots".

17 Aus Sicht der Völkerrechtslehre in der VR China geht lediglich die Ausübung der Souveränität über, da das Völkerrechtssubjekt China in den ungleichen Verträgen die Souveränität rechtlich nicht wirksam habe abtreten können.

18 Siehe dazu: Wolfgang Mincke, "Die Problematik von Recht und Sprache in der Übersetzung von Rechtstexten", Archiv für Rechts-und Sozialphilosophie, Vol. 1991 LXXVI, S. 446-465.

19 Optimistisch-pragmatisch: D. Roebuck / Sin, King-kui, "The Ego and I and Ngo - Theoretical Problems in the Translation of the Common Law into Chinese"; Working Papers in Languages and Linguistics; (1992) 3; City Polytechnic of Hong Kong; pp. 1-47. - Pessimistisch-f unktional: $E$. Epstein, "China and Hong Kong: Law, Ideology and the Future Interaction of the Legal Systems", in: Raymond Wacks (ed.), The Future of the Law in Hong Kong, Hongkong 1989, pp.61-63. 
sollen Terminologie und Stil der Rechtssprache in der Volksrepublik China, der Republik China auf Taiwan, der portugiesischen Ubersee-Region Macao und dem Kaiserreich Japan, die alle etwas vereinfachend der Civil Law-Rechtsfamilie zugerechnet werden können, zum Vergleich herangezogen werden.

\section{Bedeutungszuwachs der chinesischen Sprache im Rechtsleben Hongkongs}

\section{a. Frühkoloniale Dominanz der englischen Rechtssprache (1842 - 1933)}

Durch die Unterzeichnung des ungleichen Vertrages20 von Nanking am 29.8.1842 trat das chinesische Kaiserreich die bis dahin unbedeutende Insel Hongkong am Eingang des Perlflusses in Südchina an das Vereinigte Königreich von Großbritannien und Nordirland ab.21 In weiteren Verträgen erzwang die britische Krone von der regierenden Qing-Dynastie (1644-1911) die Abtretung der Halbinsel Kowloon22 und nötigte sie zu einem 99-jährigen Pachtvertrag bezüglich der Neuen Territorien23. Die vor Ankunft der Briten weitgehend aus Fischern, Piraten und Bauem bestehende Bevölkerung Hongkongs war nur in ganz groben Zügen mit dem kaiserlich chinesischen Recht vertraut.24 Ihre Beziehungen regelten sie meist außerrechtlich.

20 Chin.: Bu pingdeng diaoyue [engl.: "unequal treaty"]. Die politische und völkerrechtliche Theorie der VR China erkennt die Gültigkeit des infolge der Niederlage Chinas im sog. Opiumkrieg vom britischen Empire erzwungenen Abtretungsvertrages nicht an, obowhl sämtliche chinesischen Regierungen seither de facto Großbritannien als für Hongkong verantwortlichen Gesprächs- und Vertragspartner akzeptien haben. Großbritannien rechtfertigt die Gültigkeit dieses in China zu Recht als ungleich bezeichneten Vertrages damit, daß nach dem seinerzeit geltenden Völkerrecht eine erzwungene Abtretung von Gebieten als Kriegsreparation auch für imperialistische Kriege erlaubt war. Fraglich ist jedoch, ob das intertemporale Völkerrecht hier hicht durch naturrechtliche Grundsätze durchbrochen wird. Seit Unterzeichnung der Germeinsamen Erklärung hat sich diese Kontroverse zunächst entschärft, wenngleich sie nach 1997 durchaus wieder aufflammen könnte, wenn Großbritannien von China die Einhaltung von Menschenrechten in Hongkong aufgrund der Gemeinsamen Erklärung verlangt und China dies als Einmischung in innere Angelegenheiten zurückweist.

21 Der Austausch der Ratifikationsurkunden erfolgte am 26.6.1843, worauf Sir Henry Pottinger aum ersten Govemor Hongkongs emannt wurde. Siehe: GB. Endacott, A History of Hong Kong , London 1958, p. 22.

22 Convention of Peking, Oktober 1860; G B. Endacott, a.a.O. p. 110.

23 Am 1.7.1898 begann die Laufzeit des Pachtvertrages, dessen Auslaufen am 30.6.1997 mit dem Souveränitätswechsel in Hongkong koinzidiert; GB. Endacott, a.a.O. p. 262.

24 Dazu: EJ. Eitel, Europe in China, Hongkong/London 1895, pp. 127-134. 
Die Briten brachten mit dem Common Law 25 auch die englische Rechtssprache in die Kronkolonie Hongkong.26 Zunächst sahen die Instruktionen aus London vom 3.6.1843 vor, $\mathrm{da} B$ die chinesischen Bewohner Hongkongs, vorbehaltlich einer ordre public-Klausel, nach chinesischem Recht und Brauchtum zu beurteilen seien.27 Als unpraktikabel und politisch unerwünscht erwies es sich, die chinesischen Bewohner Hongkongs chinesischer Gerichtsbarkeit zu unterstellen. 28 Aufgrund von lokalen Gesetzen (ordinances) aus dem Jahre 1844 wurde dem Obersten Gerichtshof (Supreme Court) und den Untergerichten (magistrates) die Jurisdiktion auch über die chinesische Bevölkerung Hongkongs zuteil.29 Durch Erörterungen über das zunächst durch prärogative Gesetzgebung (prerogative legislation), lokale Gesetzgebung und lokale Rechtsprechung, später durch die Anwendung britischer Parlamentsgesetze (Acts of Parliament) und britischen Fallrechtes (case law) ${ }^{30}$ mehr und mehr verdrängte chinesische Gewohnheitsrecht, das bis heute als subsidiäre Rechtsquelle in Hongkong gilt, 31 wurden einige kaiserzeitlich-chinesische Rechtsinstitute den englischsprachigen Juristen zugänglich. Daneben gab es bis in das Jahr 1962 hinein Kuriositäten wie die eines "special magistrate"32, der Entscheidungen pragmatisch an chinesischen Gewohnheiten ausrichtete. Zusammenfassend bleibt festzustellen: Bei der Anwendung von traditionellem chinesischen Recht bereicherte man zwar die englische Rechtssprache um chinesische Rechtskonzepte, aber umgekehrt wurde keine chinesische Common Law-Terminologie geschaffen.

25 Common Law i.e.S. und Equity gelten nur aufgrund der Application of English Law Ordinance 1856, No. 7, cap. 88. Zur 1966 erfolgten Gesetzesänderung bezüglich der Interaktion zwischen vor dem Stichtag 5.4.1843 geltender Acts of Parliament und dem Common Law i.e.S., siehe: Peter Wesley-Smith, "The Effect of Pre-1843 Acts of Parliament in Hong Kong", Hong Kong Law Journal (1984), p. 142.

26 Siehe die demnächst erscheinende juristische Dissertation von Kentaro Matsubara, "Chinese Legal Tradition in its Interaction with the Reception of British Laws in Hong Kong" (working title), St. Antony's College/Oxford.

27 Siehe: GB. Endacott, a.a.O. pp. 38-41. - Im Jahre 1851 wurde eine Art polizeilicher Selbstverwaltung für Chinesen eingeführt, die sich jedoch nicht bewährte und später einschlief.

28 Siehe dazu die Auführungen bei: $G$ B. Endacott, a.a.O. pp. 40, 41, 57, 58.

29 G B. Endacott, a.a.O. p. 58.

30 Application of English Law Ordinance, 1856, No. 7, (cap. 88).

31 Zusammenfassend: Chinese Law and Custom in Hong Kong, Report of a Committee appointed by the Govemor in October 1948, Govemment Printer 1953. Stichtag für die letze Anerkenntnis des Eingehens familienrechtlicher Sonderinstitute (Konkubinen = "chip" oder "tsip", Adoption) im chin. Gewohnheitsrecht Hongkongs ist der 7.10.1971. Siehe auch: Peter Wesley-Smith, "The Legal System and Constitutional Issues", in: Wesley-Smith / Chen (eds.), The Basic Law and Hong Kong's Future, Hong Kong 1988, p. 177. Weiterhin: J. Hayes, "Chinese Custornary Law in the New Territories of Hong Kong. The Background of the Operation of the New Territories Ordinance, 1899-1987", in: Asian Profile (Hong Kong), 19 (April 1991) 2, pp. 97-136.

32 Siehe dazu: Austin Coates, "Myself a Mandarin - Memoirs of a Special Magistrate", Hongkong 1968. 
Am 23.3.1858 wurde in der Hongkong Government Gazette Extraordinary erstmalig ein lokales Gesetz (ordinance) auch in chinesischer Version veröffentlicht. 33 Abgesehen von den Berichten über Kriminalfälle in der chinesischsprachigen Tagespresse ${ }^{34}$ und der außergerichtlichen Schlichtung von Zivilrechtsdisputen durch chinesische Schlichter 35 kam es jedoch nicht zur Herausbildung einer spezifisch Hongkong-chinesischen Rechtssprache. Dies belegen auch die ersten in Hongkong veröffentlichten Englisch-Chinesischen und Englisch-Kantonesischen Wörterbücher, deren Rechtswortschatz kümmerlich ist. 36

Als erster chinesischer Geschworener in der Geschichte Hongkongs wurde am 24.2.1858 Wong A Shing vom Legislativrat (Legislative Council) 37 bestätigt. 38 In seiner Funktion als Geschworener mußte auch er sich der englischen Sprache bedienen. Bei seinem späteren Beruf als Dolmetscher am Supreme Court beschränkte sich sein chinesisches Vokabular eher auf tatsächliche, denn auf rechtliche Begriffe. Selbst von den berühmten im Common Law ausgebildeten Hongkonger Juristen $\mathrm{Ng}$ Choy 39 und Ho Kai40 sind keine Anstrengungen bekannt, das Common Law in chinesischer Sprache zugänglich zu machen. Die englische Sprache war der Schlüssel zu Prestige und Karriere für die Hongkong-Chinesen. Dies galt insbesondere für den eng mit der Kolonialmacht verbundenen rechtlichen Bereich. Im nichtkolonialisierten, aufgeschlossenen Japan hatte Hijikata Yasushi an der Kaiserlichen Universität Tokyo (jetzt: Universität Tokyo) schon im Jahre 1887 ein Lehrbuch über das

33 "An Ordinance for Licensing and Regulating the Sale of Prepared Opium", Ond. No. 2/17.3.1858; in: Hongkong GovemmentGazette Extraordinary, p. 3.

34 Z.B.: Xunhuan Ribao (Cheungwaahn Yahtbou), seit 1874, Hrsg.: Wang Tao.

35 Siehe: $G$ B. Endacott, a.a.O. pp. 84-85.

36 Z.B.: William Lobscheid, A Chinese and English Dictionary, Hongkong 1871. Tam, Tat-hin, An English and Chinese Dictionary with English Meaning or Expression for every English Word, Hongkong 1875.

37 Nicht demokratisch legitimiertes Beschlußfassungsorgan, das sich seinerzeit aus ex-officio Mitgliedem der Kolonialregienung und aus vom Govemor emannten Mitgliedem zusammensetzte.

38 Siehe: James W. Norton-Kyshe, The History of the Laws and Courts of Hong Kong, Hongkong 1898 , p. 465.

$39 \mathrm{Ng}$ Choy (1842-1922), geboren in Singapur, ausgebildet in England, wurde 1877 als Mitglied des Lincoln's Inn zum ersten chinesischen barrister emannt. 1880 wurde er zwischenzeitlich stipendiary magistrate in Hongkong. Govemor Sir John Hennessy berief ihn 1880 zum ersten chinesischen Legislative Councillor in der Geschichte Hongkongs. Unter dem Namen Wu T'ing-fang trat er später in kaiserlich-chinesische Dienste ein, war Vertrauter Li Hung-changs und vertrat China als Botschafter in den U.S.A. (1896/97). Späternahm er als Anhänger Sun Yat-sens wichtige Positionen in der Regierung der Republik China ein. Siehe: $G B$. Endacolt, Govemment and People in Hong Kong 1841-1962 - A Constitutional History, Hongkong 1964, pp. 92-93.

40 Ho Kai (1859-1914), verschwägert mit Ng Choy, war in England zum barrister und Ar $\pi$ ausgebildet worden. 1884 wurde er Legislative Councillor. Nach dem Tode seiner englischen Ehefrau, Alice, erwarb er sich Verdienste bei der Einrichtung eines Krankenhauses und des Hong Kong College of Medicine (1887). Siehe: G B. Endacott, A History of Hong Kong, London 1958, p. 249; John K. Fairbank (ed.), The Cambridge History of China, vol. 10, p. 584. 
englische Vertragsrecht veröffentlicht, wobei er sich meist chinesischer Schriftzeichen (Kanji) für die Ubersetzung der Common Law-Termini bedient hatte. 41

\section{b. Anfangsgründe einer chinesischen Common Law-Terminologie (1934-1973)}

1934 übersetzte man an der Hongkonger Chinese General Chamber of Commerce die Companies Ordinance (Amendment). 42 Etwas später erschien das beeindruckende, aber nichtoffizielle Übersetzungswerk des Ma Yuen43. Ma Yuens Übersetzung der Hongkonger ordinances aus dem Jahre 1936 stellt eine Pioniertat auf dem Gebiet der chinesischen Common Law-Rechtsterminologie in Hongkong dar.

Etwa zur gleichen Zeit wuchs auch in China das Interesse am Common Law, obwohl man sich dort für die Rezeption des kontinentaleuropäischen Civil Law entschieden hatte.44 Es erschienen chinesische Übersetzungen der Standardwerke im anglo-amerikanischen Vertragsrecht von Sullivan45 (1933) und Holland46 (1945). Die Law School der Soochow Universität in Schanghai, an der man Erfahnungen mit den gemischten Gerichten (Mixed Courts) in den internationalen Konzessionen auswertete, galt als Zentrum der Common Law-Anhänger in China. Aber auch hier bediente man sich vorzugsweise der englischen Sprache. 47 Eine Interaktion mit der chinesischen Rechtssprache in Hongkong ist nicht nachweisbar.

41 Hijikata Yasushi, Eikoku keiyakuhou [engl.: "English Law of Contract"], Tokyo 1887.

42 Xianggang Gongsi Zeli (Heunggong Gungsi Jaklaih), Xianggang Huashang Zonghui (Heunggong Wahsaang Jungwuih), Hongkong 1934.

43 Ma Yuen (transl.), Ordinances of Hongkong: 1844 to 1936, 2 vol., Overseas Chinese Daily News (publ.), Hongkong 1936.

44 Siehe: Karl Bünger, "Die Rezeption des Europäischen Rechts in China", in: Ernst Wolff (Hrsg.), Beiträge zur Rechtsforschung, Berlin/Tübingen 1950, S. 166-189.

45 Meiguo Qiyuefa Tonglun [engl.: "A General Survey of American Contract Law"], Übersetzung des 1.Teils des Werkes "American Business Law" von John J. Sullivan durch Huang Meiqing, Lingen University, Kanton/China 1933.

46 Yingguo Qiyuefa, Übersetzung des Werkes "English Law of Contract" von Holland durch Lin Zhenkang, China 1945.

47 In der von der Law School herausgegebenen Publikation "Dongwu Fasheng" [engl.: "Eastem Wu Voice of Law"], finden sich jedoch einige chinesischsprachige Artikel über die Grundzüge des Common Law, z. B.: 1944, Heft 2, S. 2 ff. und 1947, Heft 1, S. 7 ff. Demnächst ist eine rechtsgeschichtliche Untersuchung über die Law School der Soochow Universität zu erwarten: Alison Conner, University of Hong Kong, Faculty of Law. 
Am 31.5.1940 erschien die erste chinesischsprachige Ausgabe der Hongkong Government Gazette48. Die Verwendung einer japanisch-chinesischen Rechtssprache während der japanischen Besetzung Hongkongs war zu kurz, um sich auf das Common Law auszuwirken. 49

Die Nachkriegszeit erlebte eine auf den Stand von 1953 gebrachte, verbesserte Neuauflage von Ma Yuens Ưbersetzungswerk. 50 Weiterhin kam es im politischen Bereich zu einem erstmals auch in chinesischer Sprache abgefaßten offiziellen Untersuchungsbericht 51 über Unruhen in Kowloon und Tsuen Wan im Jahre 1956 und im juristischen Bereich zur Kommentierung einiger im kommerziellen Sektor wichtigen Vorschriften. 52

\section{c. Erhebung des Chinesischen zu einer Amsssprache (1974-1986)}

Erst in den siebziger Jahren änderte sich jedoch der rechtliche Status des Chinesischen. Auslöser dafür waren die durch die Kulturrevolution und soziale Probleme ausgelösten gewalttätigen Unruhen 53 besonders der Jahre 1966/67, die die sonst eher politisch passiven Hongkonger Universitätsstudenten zu einer Kampagne für die Erhebung des Chinesischen zu einer Amtssprache veranlaßten. Diese Kampagne erhielt aufgrund der allgemeinen Besorgnis über die gefährdete Stabilität Hongkongs und wegen der offensichtlichen Ungerechtigkeit, 98\% der Bevölkerung die Sprache der Kolonialmacht aufzudrängen, die Unterstützung weiter Bevölkenmgskreise.

Um die gespannte Lage zu beruhigen, setzte die Hongkonger Regierung im Oktober 1970 das Chinese Language Committee ein, welches zwischen Februar und Juli 1971 vier

48 G B.Endacott, A History of Hong Kong, London 1958, p. 113, spricht von einer chinesischen Version der Govemment Gazette schon im Jahre 1860, die neben offiziellen Informationen und Erklärungen auch Berichte über interessante Rechtsfälle und europäische Kurmachrichten enthalten habe. Eine solche Version ist in Hongkong nicht auffindbar.

49 Siehe z.B.: Xiangdu Ling Teji (Heungduk Lihng Dahkchap), [dtsch: "Sonderausgabe der Befehle des Oberkommandierenden in Hongkong"], Hongkong, Shouwa Jahr 18 (=1943).

50 Ma Yuen (transl.), Ordinances of Hongkong: 1844 to 1953, 4 vol., Overseas Chinese Daily News (publ.), Hongkong 1953.

51 Jiulong ji Quanwan Baodong Baogaoshu (Gauluhng kahp Chyuhnwaan Bouhduhng Bougousyu), Xianggang Zongdu Cheng Zhimindibu Dachen Han (Heunggong Jungduk Chihng Jihkmahndeihbouh Daaihsahn Haahm), [dtsch: "Bericht des Govemors von Hongkong an den Minister des Collonial Office"], Hongkong 1956.

52 Z.B.: Jin Li (Gam Lihk), Xianggang Waibi Guanzhi Guize Xinbian (Heunggong Ngoihwuih Gunjai Kwaijak Sanpin), [engl.: "New Explanation of the Hong Kong Foreign Exchange Control Regulations"], Hongkong 1963.

53 Siehe: Kowloon Disturbances 1966 - Report of the Commission of Inquiry, Hong Kong Govemment Printer, 1969. 
Berichte veröffentlichte. ${ }^{54}$ Die weitreichenden Empfehlungen dieser Berichte zur Aufwertung des Chinesischen fanden nur zum Teil Eingang in die 1974 beschlossene Official Languages Ordinance.55 Danach wurden Englisch und Chinesisch zu offiziellen Sprachen Hongkongs zum Zwecke der Kommunikation zwischen der Regienung bzw. einer ihrer Beamten und der Offentlichkeit.56 Den beiden Amtssprachen kommt in dieser Funktion gleicher Status und "equality of use"57 zu. Verantwortlich für die Umsetzung wurde die Chinese Language Division of the Home Affairs Branch of the Govemment Secretariat 58 . In der Praxis werden seither alle Bekanntmachungen, Berichte (z.B. von Untersuchungsausschüssen) und Planungen (green and white papers) zweisprachig erstellt. Auch Formulare und Lizenzen werden in zweisprachiger Form ausgegeben. Reden im Legislative Council, dem quasi-Parlament Hongkongs, dürfen in Kantonesisch gehalten werden,59 woraufhin sie im Protokoll in chinesischer Schriftsprache festgehalten werden. Ein Simultanübersetzungsdienst wird auch im Urban Council, in den District Boards und bei Pressekonferenzen der Regierung angeboten.60

Die Official Languages Ordinance (1974) beendete ihrerzeit noch nicht die Dominanz der englischen Sprache in der Gesetzgebung. Fortschritte gab es jedoch bei der Zulassung des Chinesischen als Sprache des Gerichtsverfahrens. Während das Verfahren im Privy Council, im Court of Appeal, in den High Courts und in den District Courts weiterhin in englischer Sprache durchgefühn werden mußte, wurde den Magistrates' Courts, den Labour Tribunals, den Small Claims Tribunals, den Juvenile Courts, den Immigration Tribunals und den Coroners das Recht eingeräumt, sich vor Prozeßbeginn auf das Englische oder das Chinesische (Kantonesische61) als Verfahrenssprache festzulegen.62 Das Gerichtsprotokoll sollte weiterhin in englischer Sprache geführt werden, um den meist nur Englisch sprechenden Richtem der Obergerichte ein etwaiges Berufungsverfahren zu erleichtern63.

54 The First, Second, Third and Forth (and Final) Reports of the Chinese Language Committee, Hong Kong Government Printer, 1971.

55 Cap 5, LHK (Laws of Hong Kong) 1980 ed.

56 Official Languages Ordinance (1974), section 3(1).

57 Official Languages Ordinance (1974), section 3(2).

58 Zur Atheit dieser Abteilung siehe: F. Ting (Commissioner for Chinese Language), An Introduction to the Work of the Chinese Language Division, (1984) 4 Ming Pao Monthly, p. 13 (in Chinesisch).

59 Mandarin ist gem. order 2(1) der standing orders des Legislative Councils (app. I, LHK 1971 ed.) (noch!) nicht augelassen.

60 Siehe: F. Ting, South China Morning Post, Nov. 7, 1983 - zitien bei: Albert Chen, "1997: The Language of the Law in Hong Kong", Hong Kong Law Joumal (1985), No. 1, p. 22, Fn. 17.

61 So jedenf alls die Empfehlung des Chinese Language Committees in seinem Third Repon, para 22 24

62 Official Languages Ordinance (1974), section 5 with schedule.

63 Die Praxis folgt seither der Empfehlung des Chinese Language Committee, Third Repon, para 26 (vii). 
Ladungen, Klagformeln (writs) und Bekanntmachungen der Gerichte wurden zweisprachig. 64

Mit der Official Languages Ordinance (1974) sprach sich der Gesetzgeber damals ausdrücklich gegen eine zweisprachige Gesetzgebung aus.65 Die Chinese Language Division hat trotzdem seither wichtige ordinances versuchsweise ins Chinesische übersetzt. 66 Diesen zum Teil ohne die englischsprachige Originalvorlage schwer verständlichen Übersetzungen67 kommt keine offiziell bindende Geltungskraft zu.68 Eine Ubersetzung des case law, das aus Common Law i.e.S. und equity besteht, wurde seinerzeit nicht in Angriff genommen. 1975 erschien ein von der Regierung gefördertes, aber nichtoffizielles EnglischChinesisches Rechtsglossar.69

\section{d. Aufbau eines zweisprachigen Rechtssyst ens (seit 1987)}

Erst nach der Unterzeichnung der Gemeinsamen Erklänmg der Regierung des Vereinigten Königreiches von Großbritannien und Nordirland und der Regierung der Volksrepublik China am 26.9.1984, die den Souveränitätsübergang bezüglich des Gesamtterritoriums der Kronkolonie für den 1.7.1997 vorsieht und in der den Hongkongern unter der vagen Formel "ein Land, zwei Systeme" unter anderem ein 50-jähriges Fortbestehen des Common LawRechtssystems versprochen wurde70, machte man sich in Hongkonger Regierungskreisen neue Gedanken darüber, wie das Fortbestehen des Common Law in Hongkong für die Zeit nach 1997 am besten zu sichern sei.

64 Siehe: Albert Chen, a.a.O. p. 26.

65 Official Languages Ordinance (1974), section 4(1).

66 Die Publikation dieser Übersetzungen im strafrechtlichen, familienrechtlichen, arbeitsrechtlichen, verfassungsrechtlichen und wirtschaftsrechtlichen Bereich erfolgte seit 1984 durch den Govemment Printer.

67 Dies liegt zum Teil an der zu engen Orientierung an der in altmodischem Englisch verfaßten, schon für sich alleine - ohne Kenntnis des einschlägigen case law - schwer verständlichen Originalvorlage. Vgl. z.B. die Übersetzung der "Offences against the Person Ordinance", cap 212 , section 6, Chinese Language Division, Govemment Printer, 1985.

68 Siehe auch: Secretary of Home Aff airs, LegCo Proc 1983-84, 1178 (Aug.10, 1983).

69 Glossary of Applied Legal Terms (English-Chinese), entstanden in Kooperation zwischen Regierung und Experten an der Chinese University, Govemment Printer 1975.

70 Joint Declaration, Kap. 3, Abs. 3, S. 2 und Annex 1, Kap. II, Abs. 1. 


\section{Zweisprachige Gesetzgebung}

Erste Anzeichen für ein geplantes zweisprachiges Rechtssystem waren der Tagespresse zu entnehmen.71 Sie bezogen sich auf die Schaffung eines zweisprachigen statute law. In seiner Eröffnungsansprache an den Legislative Council am 30.10.1985 gab der Govemor bekannt, daß der Executive Council 72 im Juli 1985 beschlossen habe, authentische chinesische Versionen des Hongkonger statute law zu erstellen.73 Daraufhin setzte der Attomey General74 im September 1985 eine Arbeitsgruppe75 ein, deren Bericht76 im April 1986 veröffentlicht wurde. Dieser Bericht führte zu der Verabschiedung der Official Languages (Amendment) Ordinance 77 und der Interpretation and General Clauses (Amendment) Ordinance78 an 25.3.1987. Die Voraussetzung dafitr hatte de jure die britische Krone, de facto die Regierung in London bereits am 22.8.1986 durch Erlaß der Hong Kong Additional Instructions (1986) ${ }^{79}$ geschaff en.

Die Official Languages (Amendment) Ordinance sieht im wesentlichen vor, daß beginnend mit ihrem ersten Geltungstag alle neuen ordinances, die nicht nur alte ordinances abändem, zweisprachig verabschiedet werden müssen. 80 Bezüglich der alten ordinances führt sie aus, daß der Governor-in-Council81 deren von der Law Drafting Division erstellte chinesische Versionen nach Beratung mit dem aus ehrenamtlichen Experten einzurichtenden Bilingual Laws Advisory Committee und nach Zustimmung durch den Legislative Council 82 für authentisch erklären könne.83 Die Interpretation and General Clauses (Amendment) Ordinance erklärt die chinesische und englische Version obiger zweisprachiger Ordinances für gleich authentisch. Der Governor-in-Council kann nach Zustimmung durch den Legis-

71 Bericht über eine entsprechende Initiative der Bar Association und der Law Society in: South China Moming Post, Oct. 26, 1984. Pläne des Attomey Generals in: South China Moming Post, Oct. 31, 1984 und Nov. 9, 1984.

72 Vom Govemor emanntes Gremium mit kabinettsähnlicher, beratender Funktion.

73 Siehe: Discussion Paper on the Laws in Chinese, Attomey General's Chambers, Hongkong, April 1986, p. 2.

74 Entspricht einem Justizminister mit staat sanwaltlichen Auf gaben.

75 Working Party in the Law Drafting Division of the Attomey General's Chambers.

76 Siehe: Discussion Paperon the Laws in Chinese, a.a.O.

77 Cap. 5, LHK 1988 ed.

78 Cap. 1, LHK 1989 revised ed.

79 Siehe: Michael Thomas (fomer Attomey General), "The Development of a Bilingual Iegal System in Hong Kong" , in: Hong Kong Law Joumal 1988, No. 1, p. 18.

80 Sec. $4(1), 4(2)$

81 Wenn vom Govemor in Council die Rede ist, so bedeutet das gem. der Gesetzesdefinition in sec. 3 der Interpretation and General Clauses (Amendment) Ordinance (1987), daß die Handlungen des Govemor nur durch eine Mitwirkung des Executive Council, der aber dafür nicht unbedingt einberufen werden muß, Gültigkeit erlangen.

82 Diese ist gern. sec. 4B (4) Official Languages (Amendment) Ordinance (1987) erforderlich.

83 Sec. 4B (1), 4C Official Languages (Amendment)Ordinance (1987) 
lative Council ${ }^{84}$ durch Bekannmachung in der Govemment Gazette Rechtstermini einer Sprache als gleichbedeutend mit Rechtstermini der anderen Sprache festsetzen. 85 Im Zweifel sollen folgende Interpretationsregeln gelten: $\mathrm{Zu}$ bestimmen sei "the meaning which best reconciles the text, having regard to the object and purposes of the ordinance." 86 Außerdem habe bei der gerichtlichen Interpretation von zweisprachigen ordinances die der englischen Version gegenübergestellte Common Law-Bedeutung eines chinesischen Rechtsbegriffes im Zweifel Vorrang vor anderen chinesischen Bedeutımgen dieses Rechtsbegriffes. 87

In der durch die Verzögerung der Inkraftsetzung der Official Languages (Amendment) Ordinance erwirkten Testphase (dummy run period) der Jahre 1987/88 wurden fünf neue ordinances 88 in zweisprachiger Form verabschiedet, deren chinesische Version allerdings noch nicht authentisch ist.89 In der Folge wurde der 7.4.1989 zum Stichtag für eine nun zwingend zweisprachige Gesetzgebung bestimmt. 90 Seither müssen alle neuen ordinances, abgesehen von eilbedürftigen Notfallverordnungen 91 in englischer und chinesischer Sprache erstellt werden, wobei beide Versionen von gleicher Authentizität sind. Bis zum 30.11. 1992 waren bereits 61 zweisprachige Versionen von neuen ordinances oder von Gesetzesänderungen (amendments) existierender zweisprachiger Versionen in der Govemment Gazette veröffentlicht worden.92

Lange Zeit wurde hingegen die Übersetzung der für die Schaffung eines zweisprachigen statute law wesentlich wichtigeren älteren ordinances nicht ernsthaft in Angriff genommen. Die sozialen und politischen Unruhen in China, die am 4. Juni 1989 in Peking blutig niedergeschlagen wurden, gaben dem ProzeB der Demokratisierung und Politisierung Hongkongs neue Nahnmg, so daB auch die Forderungen nach Recht in der Muttersprache wieder lauter wurden. Der vehementeste Verfechter eines legal bilingualism in Hongkong war Albert H.Y. Chen von der Rechtsfakultät der University of Hong Kong93. Seine Kritik

84 Sec. 10E (2) Interpretation and General Clauses (Amendment) Ordinance (1987)

85 Sec. 10B (1), 10B (2), 10E (1)Interpretation and General Clauses (Amendment) Ordinance (1987)

86 Sec. 10B(3) ist der völkerrechtlichen Auslegungsregel in Art. 33 IV der "Wiener Vertragsrechtskonvention" vom 23.5.1969 nachgebildet. Siehe: United Nations Conference on the Law of Treaties, Off. Rec., UN Doc.A/CONF. 39/11/Add. 2, New York 1971, p. 287.

87 Sec. 10C (1). Unter Common Law ist hier gem. sec. 10C (2) das Fallrecht zu verstehen.

88 Siehe Aufstellung bei: Albert Chen, "The Bilingual Legal System in Hong Kong: A Gloomy Future", in: Hong Kong Law Joumal 1991, No. 1, p. 16, Fn. 8.

89 Siehe: Michael Thomas, a.a.O. p. 19.

90 Siehe: Albert Chen, a.a.O. p. 15.

91 Official Languauges (Amendment) Ordinance (1987), sec. 4 (3).

92 Neue ordinances (+amendments neuer ordinances): ab 7/4/1989: 8, 1990: $22(+2), 1991: 14(+3)$, bis 30/11/1992: $10(+2)$.

93 Siehe folgende Publikationen Albert H.Y. Chens zum Thema: (1) "1997: The Language of the Law in Hong Kong", Hong Kong Law Joumal 1985, No. 1, pp. 19-47; (2) "Language, Law and the Case of Hong Kong", Paper for a Conference on the Common Law in Asia, University of Hong 
an dem schleppenden Tempo der Úbersetzung alter ordinances und einer falschen Prioritätensetzung 94 schien jedoch unter der Kolonialregierung des vorsichtigen Sir David Wilson auf taube Ohren zu stoßen. Während Sir David Wilson sich nach dem 4. Juni 1989 sehr zögerlich bezüglich der Verankerung demokratischer Strukturen verhalten hatte, fährt der neue Govemor, Mr. Christopher Patten, seit seinen kontroversen Vorschlägen zur Wahlrechtsreform95 am 7.10.1992 mit Rückendeckung aus London einen mutigeren Kurs. Abzuwarten bleibt, welche Folgen dies für den legal bilingualism haben mag. Die in der Govemment Gazette vom 24.7.1992 veröffentlichte chinesische Version der Interpretation and General Clauses Ordinance stellt die erste für authentisch erklärte Übersetzung einer älteren ordinance dar.96 Bei der für ein funktionierendes zweisprachiges Rechtssystem entscheidenden Frage der Übersetzung fundamentaler alter ordinances scheinen Law Drafting Division und Bilingual Laws Advisory Committee seither mit Riesenschritten vorangekommen zu sein.97 Zum verzögernden Element sind neuerdings der mit der Tagespolitik vielbeschäftigte Legislative Council und sein besonderer Ausschuß (Ad hoc committee) geworden. Im Vergleich zur Übersetzungstätigkeit in Macao, die in einem überschaubaren Expertenrahmen von statten geht,98 gibt es in Hongkong zu viele Akteure. 99 Trotzdem ist die Verabschiedung der chinesischen Versionen wesentlicher strafrechtlicher alter ordinances für den Herbst 1993 zu erwarten. 100 Für die wichtigsten alten zivil-und wirtschaftsrechtlichen ordinances ist die Authentizitätserklänmg der chinesischen Versionen für das Jahr 1994 vorgesehen. 101

Kong 15.-17.12.1986. (3) "Law in a Foreign Language: The Case of Hong Kong", in: K. CheekMilby / M. Mushkat (eds.), Hong Kong: The Challenge of Transformation, Centre of Asian Studies, University of Hong Kong 1989, p. 212. (4) "The Bilingual Legal System in Hong Kong: A Gloomy Future", Hong Kong Law Journal 1991, No. I, pp. 14-18.

94 Siehe Fn. 93, (4); pp. 16-17.

94 Abgedruckt in: Electoral Arrangements for 1994-95, Compendium of Proposals, Hong Kong Govermment Printer, Jan. 1993, pp. 421-433. Ebenso: Electoral Provisions (Miscellaneous Amendments) Bill 1993, H.K. Govemment Gazette, Legal Suppl. No. 3 vom 12.3.1993.

96 Die im Jahre 1988 veröffentlichte chinesische Version der älteren englischen Version der Official Languages Ordinance wurde in der sog. dummy run period erstellt. Sie ist nicht authentisch.

97 Dies wurde aus zwei Gesprächen mit Mr. Tony Yen, Deputy Principal Crown Counsel in der Law Drafting Division der Attomey General's Chambers, im November 1992 und Januar 1993 deutlich.

98 Den Übersetzungen des Gabinete para a Traduçao Jurídica in Macao unter Leitung der Coordenadores Cabrita und Calado (Beratung: Prof. Heuser, Köln) kommt allerdings noch keine offiriclle Geltungskraft zu.

99 Die Beteiligung des Legislative Council bei der Festsetzung der chinesischen Tcrminologic ist aber für die Zeit nach 1997 als äußerst sinnvoll anzusehen, zumal ein zukünftiger Chief Executivc versucht sein könnte, et waigem "Vereinheitlichungsdruck" aus dem Norden nachaugeben.

100 Gespräch mit Tony Yen (Fn. 97) vom Januar 1993. Die draft versions haben bereits das Bilingual Laws Advisory Committee passien und werden nun schleppend vom Ad Hoc Committee des Legislative Council untersucht.

101 Gespräch mit Tony Yen (Fn. 97) vom November 1992. Die draft versions befinden sich teilweise noch in einer der beiden Arbeitsgruppen des Bilingual Laws Advisory Cornmittee. 


\section{Chinesisch als Gerichtssprache}

Auf dem Gebiet der Gerichtssprache gab es folgende Entwicklungen: Der damalige Chief Justice, Sir Denys Roberts, emannte am 15.1.1987 den jetzigen Chief Justice, Sir Ti Liang Yang, zum Vorsitzenden einer Working Party into Greater Use of the Chinese Language in Court and Court Procedure, deren Bericht aus dem Jahre 1988 weitgehende Empfehlungen auf dem Gebiet der Verwendung des Chinesischen oder des "geschriebenen Kantonesischen"102 als schriftlicher und des Kantonesischen als mündlicher Gerichtssprache macht. 103 In einem Memorandum vom 7.3.1990 bestimmte der Chief Magistrate den 17.4.1990 zum Stichtag für die Umsetzung obiger Empfehlungen in den Magistrates' Courts. Nachdem die von Nichtchinesen (expatriates) dominierte Bar Association ${ }^{104}$ gegen die Umsetzung dieses Memorandums beim Chief Magistrate und beim Chief Justice vorstellig geworden war, wurden die Anweisungen verzögert und traten erst am 15.10.1990 in Kraft. 105

Im Bereich des Fallrechts kam es am 15.9.1988 zu einem wichtigen Urteil des kontroversen High Court-Richters Sears, der unter extensiver Auslegung von section 5(3) der Official Languages Ordinance unter Heranziehung der ratio von section 19 der Interpretation and General Clauses Ordinance einem Chinesen die Verteidigung in der Mutterprache entgegen section 5(2)(b) der Official Languages Ordinance auch im High Court erlaubte. 106

\section{Die Chinese Digest}

Die wichtigste Rechtsquelle im anglo-amerikanischen Rechtskreis ist das aus fallrechtlichen Präzedenzien der oberen Gerichte bestehende Common Law107. Zweifellos ist es unmöglich, alle einschlägigen Fälle since times immemorial in das Chinesische zu übertragen. Um ein chinesisches Common Law i.w.S. fest im kulturellen Selbstverständnis der Bewohner Hongkongs zu verwurzeln, bleibt nur die Möglichkeit von kodifikationsartigen Zusammenstellungen 108 der wichtigsten Präzedenzien (leading cases) und auf lange Sicht

102 Sic (!): Report of the Chief Justice's Working Party into Greater Use of the Chinese Language in Courts and Cour Procedure, recommendation No. 7.

103 S.o., recommendation No. 7(i)

104 Vereinigung der Anwälte, die im englischen System vor Gericht plädieren dürfen.

105 Vgl.: Derek Roebuck, "The Badge of Shame: Law in a Foreign Language", unpublished paper presented to the Hong Kong University Chinese Law Research Team Meeting, May 1991, p. 3.

106 Gammon Building Construction Lid. v Cho Hing Yiu, Cons L. No. 9 of 1988 (= unreported judgment of Sept. 15, 1988).

107 Hier als Synonym des Fallrechtes (case law, incl. equity) gerneint.

$108 \mathrm{Vgl}$ : Restatements of the Law in den U.S.A. 
von Kodifikationen. ${ }^{109}$ Diese umstrittene 110 Erkennınis führte schließlich zum Chinese Digest-Projekt an der City Polytechnic of Hong Kong Law School. Das ehrgeizige Forschungsprojekt wurde am 15.11.1988 unter Federführung von Rechtsprofessor Derek Roebuck formuliert, woraufhin es Unterstützung von Regienungsseite fand. Das erste Kapitel des zivilrechtlichen Bandes über das Vertragsrecht dürfte nach kontroverser interner Diskussion über Teminologie und Sprachstil noch im Jahr 1993 fertiggestellt werden.

\section{Chinesische Common Law-Rechtsliteratur}

Eine chinesischsprachige Rechtsliteratur über das Hongkonger Rechtssystem im allgemeinen und das in Hongkong geltende Zivilrecht im besonderen ist seit Ende der 80er Jahre im Entstehen begriffen.111 Qualität und terminologisch-sprachliche Ausrichtung sind unterschiedlich. Die Stärke besonders der volksrepublikanisch-chinesischen Werke über das Common Law liegt mehr im einführenden Oberblick als im Detail.112 Ihre Terminologie ist wenig mit der der Law Drafting Division abgestimmt. Es gibt chauvinistische und wohlmeinende Versuche, den Hongkonger Juristen die Civil Law-Terminologie des volksrepublikanischen Rechtssystems aufzudrängen, wobei die ablehnende Haltung der meisten Hongkonger aus politischen Gründen verstăndlich ist. Die auf dem Markt befindlichen Englisch-Chinesischen Rechtswörterbücher können ein noch fehlendes offizielles Glossar der Law Drafting Division nicht ersetzen.

109 In den Common Law-Jurisdiktionen von Australien und Indien wurden das allgemeine Straf recht und das Vertragsrecht kodifiziert. Vgl.: D. Roebuck, a.a.O. p. 5.

110 Kritisch: Epstein, a.a.O.pp. 63-64, ebenso: Thomas $U$ jejski, "The Future of the English Language in the Hong Kong Law", in: $R$. Wacks (ed.), The Future of the Law in Hong Kong, Oxford 1989, pp. 180, 182-185. Optimistisch: Roebuck, a. a.O. pp. 4-5.

111 Siehe unter Quellen im 4. Kapitel.

112 Z.B.: Li Zepei (et al. Shenthen Daxue), Xianggang Falü Gaishu, [engl.: "An Outline on the Laws of Hong Kong"] Joint Publ., Hongkong 1988; Dong Liken, Xianggangf a de Lilun yu Shijian, [engl.: "Theory and Practice of Hong Kong Law"], Falü Chubanshe, Peking (?) 1990; Li Qixin (et al.), Xianggangfa Jiaocheng, [engl.: "Lectures on Hong Kong Law"], Zhongshan Daxue Chubanshe, Kanton 1991; Li Changdao (et al.), Xianggang Zhengzhi yu Fazhi, [engl.: "Political and Legal System of Hong Kong"], Shanghai Shehui Kexue Chubanshe, Shanghai 1991; Li Zepei (et al.), Xianggang Falü Da Quan, [engl.: "A Comprehensive Compendium of Hong Kong Laws"], Falü Chubanshe, Peking 1992; Zhang Xueren, Xianggangf a Gailun, [engl.: "An Outline on Hong Kong Law"], Wuhan Daxue Chubanshe, Wuhan 1992; Dong Likun (et al. Shanghai Kexueyuan), Xianggang Falü yu Sifa Zhidu, [engl.: "The Laws and the Judicial System of Hong Kong"], Zhonghua Shuju, Hongkong 1992; Zhang Zengqiang (et al.), Xianggang Fazhi Jiaocheng, [engl.: "Lectures on the Hong Kong Legal System"], Lingnan Daxue Chubanshe, Kanton 1992; Yun Guanping (et al.), Jibenfa Gailun, [engl.: "An Outline on the Basic Law"], Lingnan Daxue Chubanshe, Kanton 1992. 
Kurse über das volksrepublikanisch-chinesische Recht und seine Rechtsterminologie wurden an den Rechtsf akultäten der University of Hong Kong und der City Polytechnic of Hong Kong seit Beginn der 90er Jahre eingeführt.113 Viel wichtiger für das Erlemen der chinesischen Common Law-Terminologie ist die Tatsache, daß sich viele Erstsemester neuerdings auch über chinesischsprachige Lehrbücher einen schnellen Überblick über das fremdsprachige Recht verschaffen können.

\section{Rechtssprache und Souveränitätswechsel im Jahre 1997}

Im folgenden soll eine Prognose für die Rechtssprachensituation in Hongkong nach der britischen Souveränitätsübergabe an die Volksrepublik China am 30.6.1997 angestellt werden. Dabei ist zunächst der durch die Gemeinsame Erklärung114 und das Grundgesetzl15 für die Sonderverwaltungszone Hongkong vorgegebene rechtliche Rahmen zu beachten.

Die Gemeinsame Erklärung statuiert, daß das Hongkonger Rechtssystem noch für 50 Jahre nach der Souveränitätsübernahme in seinen wesentlichen Zügen weiterbestehen soll.116 Sie enthält zur Frage der offiziellen Sprache in der Sonderverwaltungszone die vage Bestimmung, daß neben dem Chinesischen "auch" das Englische als Sprache von Regierungsorganen und als Gerichtssprache "benutzt werden könne".117 Fraglich ist, welche Bedeutung den Worten "may also be used" oder "hai ke shiyong" in diesem Zusammenhang zukommt. Vieles wird hier von den sprachlichen Präferenzen und Loyalitäten des Rechtspersonals nach 1997 abhängen. Der schon beginnende Sinisierungsproze $B$ bei der Personalstruktur und Ausbildung des Juristenstandes mag zunächst die Zweisprachigkeit und bei späteren Juristengenerationen sogar die Dominanz des Chinesischen begünstigen. Mit Chinesisch ist aus Chinas Sicht die offizielle Sprache der VR China, Putonghua, gemeint.118 Das Kanto-

113 Siehe: Roebuck, a.a.O. p. 2.

114 Siehe: Fn. 4.; Kurztitel: Joint Declaration, Lianhe Shengming, [dtsch: "Gemeinsame Enklärung"].

115 Thonghua Renmin Gongheguo Xianggang Tebie Xingzhengqu Jibenf a [inoffizieller engl. Titel: "The Basic Law of the Hong Kong Special Administrative Region of the People's Republic of China"], vom 4.4.1990, Hongkong (Joint Publ. Co.) 1991. Kuraitel: Jibenfa (Basic Law). In Deutschland hat sich leider die irreführende Übersetzung mit "Grundgesetz" eingebürgert. Besser: "Fundamentalstatut". Englische Version abgedruckt in: China aktuell 4/1990, S. 230 ff.

116 Joint Declaration, Kap. 3, Abs. 3, S. 2: "The laws currently in force in Hong Kong will remain basically unchanged."

117 Joint Declaration, Annex 1, Kap. I, Abs. 4: "In addition to Chinese, English may also be used in organs of govemment and in the courts in the Hong Kong Special Administrative Region."

$118 \mathrm{Vgl}$. Ar. 19, S. 5 der Verfassung der Volksrepublik China vom 12.4.1982. Die analoge Anwendung der Verf assungsbestimmungen für die Gebiete mit sog. "nationalen Minderheiten"(Ar. 4, S. 7; Art. 121) kommt wohl nicht in Betracht, da Kantonesen zur "Han"-Nationalität zählen und ihre Sprache als "Dialekt" (fangyan) bezeichnet wird. 
nesische wird auch in der kantonesischen Provinz Guangdong nicht als of fizielle Hochsprache anerkannt. Großbritannien wird sich nach 1997 wohl kaum zum Anwalt des Kantonesischen, der Langzeichen oder eines feineren Stils des Schriftchinesischen machen lassen.

Anders ist die Situation bei der fehlenden Sprachenregelung für die Gesetzgebung. Eine (analoge) Anwendung der Bestimmung zur offiziellen Sprache der Regierungsorgane und Gerichte kommt in Betracht. 119 Dies könnte zunächst Zweisprachigkeit und später Dominanz des Chinesischen in der Gesetzgebung bedeuten. Großbritannien könnte argumentieren, daß die Sprache der ordinances und des case law untrennbar mit dem Rechtssystem verbunden sei und somit in diesem Bereich der zweisprachige, im Fall des case law unter Dominanz des Englischen stehende Status quo vor dem Juli 1997 für 50 Jahre eingefroren werden müsse.

Wie können nưn Srreitigkeiten bezüglich der Auslegung der Gemeinsamen Erklärung entschieden werden? Letztlich verbleiben die besseren Karten auf Seiten Chinas, da Großbritannien nach 1997 nur politische Mittel verbleiben, die Einhaltung der Gemeinsamen Erklärung zu überwachen. Die Gemeinsarne Erklärung ist zwar bei den Vereinten Nationen registriert, und die herrschende Meinung der Völkerrechtslehre mißt ihr Bindungskraft als Völkervertragsrecht zu, 120 die VR China unterwirft sich jedoch weder der Jurisdiktion des Internationalen Gerichtshof es in Den Haag, 121 noch erkennt sie an, daß Großbritannien jemals Souveränität über Hongkong gehabt habe. Für die VR China handelt es sich bei der Hongkong-Frage um eine rein innere Angelegenheit.

Das schon in der Gemeinsamen Erklärung angekündigte Grundgesetz für die Sonderverwaltungszone Hongkong, 122 das die Verfassungsstruktur Hongkongs nach dem Souveränitätsübergang im Detail regeln soll, ist ein vom Nationalen Volkskongreß erlassenes, rein innerstaatliches Gesetz gem. Art. 31 der Verfassung der Volksrepublik China. Der Nationale Volkskongre $B$ hat seinem Ständigen Ausschuß das Recht bezüglich der Auslegung des Grundgesetzes eingeräumt, welches bei dem unterschiedlichen Verfassungsverständnis in Hongkong und der VR China mit dem Interpretationsrecht des einzurichtenden Supreme Court in Hongkong kollidieren könnte.123 Der Ständige Ausschuß hat allein die chinesi-

119 Siehe Fn. 116. Dagegen spricht die sich zunehmend ausprägende Gewaltenteilung zwischen Regienung und Legislative Council, mit der China allerdings nicht einverstanden ist.

120 Vgl.: Y. C. Jao, "Hong Kong's Financial Crisis", in: Chiu, Hungdah (ed.), Symposium on Hongkong: 1997, Occasional Papers Reprints Series in Contemporary Asian Studies, No. 3, 1985. School of Law/University of Maryland, p. 8. m.w.N.

121 Eine Unterwerfungserklärung der Republik China (1946) gem. Art. 36 II des IGH-Statuts vorn 26.6.1945 liegt allerdings vor.

122 Joint Declaration, Kap. 3, Abs. 12.

123 Diesem komplexen Problem kann in dem begrenzten Rahmen dieser Arbeit nicht weiter nachgegangen werden. Siehe zu den umstrittenen Schlüsselbestimmungen der Art.19 (damals: 18) und 
sche Version des Grundgesetzes für authentisch erklärt, ${ }^{124}$ was Rückschlüsse auf die Tendenz zukünftiger Sprachpolitik zuläßt und zudem einen des Chinesischen unkundigen Richter im Supreme Court in eine peinliche Lage bringen könnte.

Als nach dem Souveränitätsübergang weitergeltende Rechtsquellen nennt Art.8 des Grundgesetzes das Common Law, die equity, die ordinances, die subsidiary legislation und das Gewohnheitsrecht. Fraglich ist, was unter Common Law zu verstehen ist 125 und wie englisch es nach 1997 noch sein darf.126 Weiterhin ist ungewiß, welcher Stichtag mit "previously in force" gemeint ist.127 Zur Rechtssprache führt das Grundgesetz aus, da $\beta$ Englisch in den Organen der Exekutive, Legislative und Judikative "auch benutzt werden könne". Englisch sei "auch eine offizielle Sprache".128 Der Rechtsausschuß des Basic Law Consultative Committee empfahl seinerzeit die Entwicklung eines zweisprachigen Rechtssystems in Hongkong. 129 Danach scheint es zunächst unwahrscheinlich, daß die Official Languages (Amendment) Ordinance 1987 und die Interpretation and General Clauses (Amendment) Ordinance 1987 am 1.7.1997 vom Ständigen Ausschuß des Nationalen Volkskongresses als unvereinbar mit dem Grundgesetz gem. Art. 160 gebrandmarkt werden. Natürlich könnte die Zweisprachigkeit zu einem späteren Zeitpunkt vom Legislative Council gem. Art. 73I Grundgesetz, zugunsten des Chinesischen abgeändert werden, ohne daß das Grundgesetz dem entscheidend entgegenstehen würde. Weiterhin käme jederzeit eine Änderung des Grundgesetzes gem. Ar. 159 Grundgesetz in Betracht, wonach die Hongkonger Sprachgesetzgebung in klaren Konflikt mit dem Grundgesetz geriete.

Zusammenf assend läßt sich wohl sagen, daß sowohl die Gemeinsame Erklärung als auch das Grundgesetz ein zweisprachiges Rechtssystem zwar zulassen, die englische Sprache

158 (damals: 169) des Grundgesetzes die Befürchtungen von Hongkongs "Mr. Democracy", Martin Lee, Chu-ming, "A Tale of Two Articles", in: Peter Wesley-Smith / Albert H.Y.Chen (eds.), The Basic Law and Hong Kong's Future, Hongkong 1988, pp. 323, 324.

124 Siehe: Beijing Rundschau 28,90, S. 5.

125 Dazu: Peter Wesley-Smith, "The Reception of English Law in Hong Kong", in: Hong Kong Law Joumal 1988, p. 183.

$126 \mathrm{Zu}$ etwaigen Änderungen der Application of English Law Ordinance vor oder nach 1997 und dem Schutz des chinesischen Gewohnheitsrechts durch die New Territories Ordinance sehr instruktiv: Peter Wesley-Smith, "The Legal System and Constitutional Issues", in: Wesley-Smith / Chen (eds.), The BasicL aw and Hong Kong's Future, Hongkong 1988, pp. 174-176.

127 Chin: "yuan you falü"; Peter Wesley-Smith, a.a.O., pp. 173-174 nennt den Souveränitätsübergang, die Verabschiedung des Grundgesetzes und die Unterzeichnung der Gemeinsamen Erklärung als mögliche Zeitpunkte.

128 Basic Law, Art. 9: "Xianggang Tebie Xingzhengqu de xingzheng jiguan, lifa jiguan he sifa jiguan, chu shiyong Zhongwen wai, hai ke shiyong Yingwen, Yingwen ye shi zhengshi yuwen." [engl.: "In addition to the Chinese language, English may also be used as an official language by the executive authorities, legislature and judiciary of the Hong Kong Special Administrative Region."]

129 Final Report on Language of the Law (passed by the Executive Committee on 8 August 1987): CCBL-SG/LES-WG01-FR02-870612(E). 
aber nicht wirksam schützen. Somit werden Sachzwänge und ethnische Zusammensetzung und Ausbildung des Rechtspersonals dazu fuhren, daß es zunächst ein zweisprachiges Rechtssystem unter Dominanz der englischen Sprache und später ein zweisprachiges Rechtssystem unter Dominanz der chinesischen Sprache in Hongkong geben wird. Ob es zu einer vollständigen "Sinisierung des Common Law" unter Absterben der englischen Rechtssprache und Isolation von den restlichen Common Law-Jurisdiktionen kommen wird, bleibt abzuwarten. Für den politisch-verfassungsrechtlichen Bereich scheint dies wahrscheinlich, für den strafrechtlichen möglich und für den wirtschafts- und privatrechtlichen schwer vorstellbar.

\section{Chinesische Common Law-Terminologie in Hongkong}

\section{a. Quellen}

Bei den Quellen chinesischer Common Law-Terminologie in Hongkong ist zwischen mündlichen und schriftlichen zu unterscheiden: $\mathrm{Zu}$ den mündlichen Quellen gehören die Gerichtssprache (Richter, barrister, Geschworene, Zeugen, Parteien), die Verwaltungs- und Parlamentssprache (Executive Council, Attomey General's Chambers, Polizei, Legislative Council, District Boards, Urban Council), die Sprache im rechtlichen Alltagsleben (solicitors 130 , Mandanten) und die im akademischen Rechtsunterricht verwendete Sprache (Lektoren, Studenten). Die mündliche Rechtssprache131 ist aufgrund ihrer situations- und personengebundenen Variabilität und Unterondnung unter die schriftliche Rechtssprache für verallgemeinerungsfähige Aussagen weniger geeignet. In Bereichen, in denen man sich um eine präzisere mündliche Rechtssprache bemüht (Gerichte, Universitäten), wäre jedoch eine nähere Analyse durchaus vielversprechend. Interessant wäre, inwieweit man auch dort auf das Phänomen des "unreinen" Chinglish132 stößt, das man ja durchaus auch als ersten Versuch der bedeutungsverlustlosen phonetischen Einverleibung fremdsprachiger Termini in das Kantonesische bewerten kann. Für die mündliche Rechtssprache ist die Frage des "brain drain" auf juristischem Sektor133 ebenso von Bedeutung wie das Problem der "Zuwanderung" (bzw. Einschleusung?) festlandchinesischer Juristen vor und nach 1997.134

130 Die Mehrzahl der Anwälte im englischen System, die den Geschäftsverkehr mit den Mandanten erledigen, sich zum plädieren vor Gericht aber eines barristers bedienen müssen.

131 Vgl. dazu die interessante mündliche Quelle der Tonbandkassetten. Z.B.: Wohng Hakminng I Faahn Mahnseuhng, Faatleuht Seuhngsik Cheuitaahm, [engl.: 'Interesting Talks about General Legal Knowledge"], Hongkong (Jauhon Cheutbaanseh) 1989.

132 Noch treffender wäre: "Canglish" (Canto-English).

133 Vgl.: The Law Society of Hong Kong, 1988 Manpower Survey of Lawyers, circular No. 182/88.

134 Siehe dazu: Ming Pao vom 18.4.1990, p. 20; Ming Pao vorn 27.4.1990, p. 30 
Im folgenden soll es jedoch vor allem um die schriftlichen Quellen gehen. Diese gliedern sich in zweisprachige Gesetzgebung135, die Chinese Digest 136, Englisch-Chinesische Rechtswörterbücher137, chinesischsprachige Lehrbücher über Aspekte des Common Law138, lokale chinesischsprachige Tageszeitungen139 und allgemeine Englisch-Chinesische Wörterbücher 140. Weiterhin kommen in Chinesisch abgefaßte Gerichtsurteile und -protokolle, zweisprachige Protokolle von Sitzungen der Legislative, zweisprachige Regie-

135 Abzustellen ist hier nicht auf die inoffiziellen chin. Versionen der ordinances, die von der Chinese Language Division erstellt wurden und beim Govemment Printer erhältlich sind. Mr. Tony Yen hat dem Autor freundlicherweise folgende vom Bilingual Laws Advisory Committee bereits abgesegnete, zur abschließenden Beratung durch den Legislative Council anstehende, chinesischsprachige Versionen, erstellt von der Law Drafting Division, zur Verfügung gestellt: Crimes Ordinance (cap. 200), Of fences against the Person Ordinance (cap. 212), Sale of Goods Ordinance (cap. 26).

136 Dem Autor wurden von Prof. D. Roebuck, Head of Law, City Polytechnic of H.K. freundlicherweise drei preliminary drafts bezüglich des Vertragsrechts zugänglich gemacht, erstellt durch lokale, bzw. festlandchinesische Experten (Dr. Sin King-kui/City Polytechnic, Prof. Theng Xianyi/Renda, Peking, Prof. Chen An/Xiamen).

137 Hongkong: William S.H. Hung, English Chinese Law Dicaionary, Chinese University, Yee Tin Tong Print. Press, Hongkong 1972; Li Choh-ming (Vice-Chancellor of the Chinese University/ supervisor) et al., Glossary of Applied Legal Terms - English-Chinese, Hong Kong Govt. Printer, Hongkong 1975; Yu Man-king, A Concise Dictionary of English Law in Chinese Translation, Southem Mat. Inc., Taipeh 1976; Chinese Language Division, Administrative Services and Information Branch, Govemment Secretariat, An English-Chinese Glossary of Terms Used in Laws of Hong Kong, Hong Kong Govt. Printer 1987; ders., An English-Chinese Glossary of Terms Commonly Used in Govemment Departments, Vol. 10 Public Order, Hong Kong Govt. Printer 1989: Li Zong'e, Everyday Law Compendium for Hong Kong, vol. 3, Comm. Press, Hongkong 1991: Yao Donghua / Ou-yang Baiquan, A Glossary of Law for Hong Kong, Comm. Press, Hongkong 1992.

VR China: He Shiying (et al.), Ying-Han Falü Cihui, [engl.: "English-Chinese Legal Vocabulary"] Beijing Daxue Chubanshe, Peking 1982; Zhang Zhongjiang (et al.), An English-Chinese Dictionary of Law, Falü Chubanshe, Shanghai 1985; Peng Jinrui (et al.), Concise English-Chinese Law Dictionary, Comm. Press, Peking/Hongkong 1991.

Taiwan: Jin Guangming, English-Chinese Dictionary of Law (Ying-Han Faxue Dacidian), Wuzhou Chubanshe, Taipeh 1988; Wunan Bianjibu, Falü Ying-Han Cidian, [engl.: "An EnglishChinese Law Dictionary"], Wunan Tushu Chuban Gongsi, Taipeh 1988; Li Qipeng, EnglishChinese Dictionary of Legal Terms (Falü Mingci Cidian), Wuzhou Chubanshe, Taipeh 1991.

Japan: Hideo Tanake (et al.), Dictionary of Anglo-American Law, Tokyo Univ. Press, Tokyo 1991.

U.S.A.: Kiang Yi-seng, An English-Chinese Glossary of American Criminal Law and Criminal Procedure Law, Cheng \& Tsui Co., Boston 1989.

138 Siehe unter c) Privatrechtsterminologie.

139 Am ergiebigsten in dieser Beziehung ist die Rubrik Fating neiwai (Faattihng ngoihnoih) [dtsch.: "Rund um das Gericht"] in der Ming Pao.

140 Z.B.: An English-Chinese Dictionary (Da Ying-Han cidian), Waiyu jiaoxue yu yanju chubanshe, Peking 1992. Weiterhin: English-Cantonese Dictionary (Ying-Yuht jihdin), New Asia - Yale-inChina Language Centre, Chinese University, Hong Kong 1991. 
rungsplanungen und -berichte und zweisprachige Formulare der Verwaltung in Betracht, die hier allerdings vernachlässigt werden sollen.

\section{b. Rechtsvergleichende Terminologiebetrachtung}

Chinesische Common Law-Terminologie, bzw. Common Law-Termini unter Verwendung chinesischer Schriftzeichen finden sich auch außerhalb Hongkongs in der Volksrepublik China, der Republik China auf Taiwan, in den südostasiatischen und überseeischen Gebieten mit chinesischem Bevölkenungsanteil und Common Law-Rechtssystem141 und in Japan.142 Die chinesische Common Law-Terminologie führt dort jedoch lediglich ein Schattendasein im Vergleich zu den gebräuchlichen offiziellen Rechtsterminologien. Trotzdem haben sich Rechtswissenschaftler in diesen Ländem aus akademischem, zunehmend auch wirtschaftlichem Interesse mit dem Studium des Common Law beschäftigt, es für eine Rezeption im größeren Stil allerdings immer wieder als ungeeignet und dem Civil Law unterlegen 143 verworfen. Eine Ausnahme stellt die an Hongkong angrenzende Sonderwirtschaftszone Shenzhen dar, die nach 1997 auch politisch an Hongkong angegliedert werden könnte, um festlandchinesischen Einfluß dortselbst zu stärken. 144

Seit der von Deng Xiaoping 1978 eingeleiteten Modemisierungspolitik nebst utilitaristischer "Offnung nach außen" besinnt sich die Volksrepublik China im Bereich des Zivilrechtes wieder auf die noch in der republikanischen Periode erfolgte, über die japanische Sprache vermittelte Rezeption des kontinentaleuropäischen 145 Zivilrechts Deutschlands und Frankreichs. Somit kann die Volksrepublik China neben der Republik China auf Taiwan, dem Japanischen Kaiserreich und der portugiesischen Ubersee-Region Macao, trotz. ciniger sozialistischer Eigenheiten, 146 von der Gesetzestechnik her der Civil Law-Rechtsfamilie zugeordnet werden. Dies gilt auch für die Dogmatik des Strafrechtes, nicht jedoch für dessen Inhalt und Anwendung. Im Strafrecht finden sich in allen Vergleichsgebieten auch noch Relikte der reichen chinesischen Strafrechtstradition.

141 Singapur, Malaysia, U.S.A., Kanada und Australien.

142 In Japan finden neben den Silbenschriften Hiragana und Katakana auch chinesische Schriftzeichen (Kanji) Verwendung, wobei die Kanji als Äquivalente für Rechtstermini bevorzugt werden.

143 Dazu: Karl Bünger, a.a.O. S. 175,176.

144 Michael Moser / Elson Pow, "Law and Investment in China's Special Investment Areas", in: $M$. Moser (ed.), Foreign Trade, Investment, and the Law in the PRC, 2nd ed. Oxford 1987, p. 20 (note 3), p. 234, sprechen von einer Super-SEZ (Super-Special Economic Zones).

145 Im "kontinentaleuropäischen Rechtskreis" spielt wiederum die Rezeption des römischen Zivilrechts eine größere Rolle als im anglo-amerikanischen.

146 Im Binnenwirtschaftsrecht findet sich noch sowjetisch inspiriertes Planwirtschaftsrecht. $7 \mathbf{u}$ den Reformbemühungen auch auf diesem Sektor: Frank Münzel, Untemehmens- und Gesellschaftsrecht der VR China, Mitteilungen des Instituts für Asienkunde, Heft 176, Hamburg 1989, S. 1-6. 
Im folgenden soll die chinesische Rechtsterminologie in Hongkong anhand von ausgesuchten Beispielen aus dem Privatrecht und Strafrecht den in den Vergleichsgebieten gebräuchlichen Rechtsterminologien gegenübergestellt werden.

\section{c. Privatrechtsterminologie}

Da bei den Grundzügen des Privatrechtes die Rechtsquelle der ordinances nur von untergeordneter Bedeutung147 ist, andererseits in den letzten Jahren auf diesem Sektor mehrere chinesischsprachige Darstellungen erschienen sind148, gibt es dort auch eine verwirrende Vielzahl von nichtoffiziellen chinesischen Common Law-Terminologieprägungen. In der Anfangsphase lehrbuchartiger chinesischsprachiger Beschäftigung in Hongkong mit den fallrechtlichen Prinzipien ist dieses Durcheinander durchaus verständlich und mitunter sogar produktiv.

Im privarechtlichen Bereich versuchen mehrere verschiedene Strömungen dem Auf einandertreffen der Hongkonger Common Law-Terminologiekonzeption mit der Civil Law-Terminologiekonzeption (besonders der Volksrepublik China) gerecht zu werden:

1. Die vorsichtigen und skeptischen "Abgrenzer", die zum Schutz des Common Law in Hongkong auch dann die Hongkonger Termini von denen der Volksrepublik China abgrenzen wollen, wenn die Rechtskonzepte beider Rechtsfamilien identisch sind. Unter den "Abgrenzern" gibt es wiederum die kantonesischen "Traditionalisten" (münd-

147 Interessant sind die Sale of Goods Ordinance (cap. 26), Limitations Ordinance (cap. 347) und Marriage Ordinance (cap. 181).

148 Vertragsrecht (law of contract): Li Zong'e, Xianggang Heyuefa yu Gongsifa (Heunggong Hahpyeukfaat yuh Gungsifaat), [engl.: "Hong Kong Contract Law and Company Law"], Comm. Press, Hongkong 1986; Yao Donghua, Hetongfa ji qi Yingyong (Hahptuhngfaat kahp keih Yingyuhng), [engl.: "Contract Law and Its Application"], Joint Publ., Hongkong 1989; He Meihuan (Betty Ho), Xianggang Hetongfa (Heunggong Hahptuhngfaat), [engl.: " The Hong Kong Law of Contract"], Chin. Univ. Press, Hongkong 1990. VA. Penlington (auth.) / Li Jiming (transl.), Xianggang Heyuefa - Fagui yu Anli (Heunggong Hahpyeukfaat - Faatkwai yuh Ngonlaih), [engl.: Hong Kong Contract Law - Statutes and Cases"], Cornm. Press, Hongkong 1992.

Deliktsrecht (law of torts): $\boldsymbol{V} \boldsymbol{A}$. Penlington (auth.) / Li Jiming (transl.), Xianggang Minshi Qinquanfa - Fagui yu Anli (Heunggong Mahnsih Chamseuhnf aat - Faatkwai yuh Ngonlaih), [engl.: "Hong Kong Law of Torts"], Comm.Press, Hongkong 1992; J.G. Fleming (auth.) / He Meihuan (transl.), Minshi Qinquanfa Gailun (Mahnsih Chamseuhnfaat Koileuhn), [engl.: "Outline on the Law of Torts"], Chin.Univ.Press, Hongkong 1992.

Stellvertretungsrecht (law of agency): He Meihuan (Betty Ho), Xianggang Dailifa (Heunggong Doihleihf aat), [engl.: "Hong Kong Law of Agency"], Chin. Univ. Press, Hongkong 1990.

Familienrecht (family law): Li Zong'e, Xianggang Jiashifa (Heunggong Gasihfaat), [engl.: "Hong Kong Family Law"], Joint Publ., Hongkong 1987. 
lich war das schon immer in Hongkong so), die mit ihnen verwandten "Sprachästheten" (das Rechtschinesisch der Volksrepublik ist zu plump), die "Kommunistenhasser" (nur so erhalten wir unser freiheitliches Rechtssystem), die "Rechtspuristen" (es gibt keine identischen Rechtskonzepte zwischen Common Law und Civil Law) und die "Lobbyisten" (wir wollen keine festlandschinesischen Juristen in Hongkong). Die "Abgrenzer" sind meist gegen eine Kodifizierung des Common Law auf dem Gebiet des Privatrechts.

2. Die "Angleicher" sind meist chinatreue "Patrioten", die aus sprachlich-kulturellem Uberlegenheitsgefühl (die Hongkonger können sowieso kein anständiges Chinesisch), Antikolonialismus (sämtliche Erinnerungen an die Kolonialheren tilgen), politischen Motiven (wider den freiheitlich-bourgeoisen Geist des Common Law) oder Opportunismus (privilegierter Repräsentant Pekings in Hongkong) heraus auch nicht davor zurückschrecken würden, Rechtskonzepte des Common Law mit Civil Law-Bezeichnungen zu versehen. Die "Angleicher" halten sich derzeit beim Privatrecht publizistisch im Hintergrund, da sie sonst in Widerspruch zur experimentellen "Ein Land, zwei (wirtschaftliche) Systeme"-Formel Deng Xiaopings gerieten. Sie tamen sich als "Harmonisierer" und warten beharrlich auf ihre Stunde.

3. Die echten "Harmonisierer", die die Rechtsterminologie Hongkongs nur dort an die der Volksrepublik China angleichen wollen, wo es die Übereinstimmung von Rechtsinstituten erlaubt. Unter den "Harmonisierem" gibt es Idealisten (demokratischer Impuls für Chinas Zukunft), Pragmatiker (nur so kann das Common Law überleben) und Opportunisten (so kann ich in der Ubergangsphase einen sicheren, oft auch lukrativen, Mittelweg gehen). Die "Harmonisierer" sind Kodifizierungen gegenüber grundsätzlich aufgeschlossen.

4. Die unbewußten "Angleicher" und "Abgrenzer", die sich mehr um eine schnelle, lukrative Publikation auf dem neuen Markt sorgen, als um die Auslotung der Unterschiede zwischen Common Law und Civil Law.

Im relativ unpolitischen, aber wirtschaftlich wichtigen zivilrechtlichen Bereich ist die Gruppe der "Harmonisierer" im Vergleich zu den "Abgrenzem" und "Angleichem" ungleich größer und facettenreicher als im straf- und verfassungsrechtlichen Bereich, wo "Harmonisierer" immer auch chinatreue "Patrioten" sind.

Ein klar abgrenzender Vergleich der Hongkonger Termini mit denen anderer Gebiete wird neben der terminologischen Unordnung in Hongkong auch dadurch erschwert, daß die ebenso unausgegorenen Ergebnisse der Common Law-Forschung in der Volksrepublik 
China ${ }^{149}$ und auf Taiwanl 50 besonders über die Quelle der Rechtswörterbücher die Hongkonger Temini nachhaltig verwirungstiftend beeinflussen. Die japanische Common LawTerminologie151 zeichnet sich dagegen durch Eindeutigkeit, Sensibilität und Klarheit aus. Ein offizielles Rechtsglossar, gestützt auf das japanische Modell, würde dieser Verwirrung in Hongkong ein Ende setzen. Im einzelnen lassen sich folgende mit Beispielen unterlegte Problemgruppen herauskristallisieren:

- Uneinheitliche Úbersetzung von Common Law-Signalwörtern:

Es gibt eine Reihe von typischen Common Law-Signalwörtem (z.B. nuisance, consideration, estoppel, privity), deren funktionelle Eigenheit eine Ubersetzung in Sprachräume mit einer Civil Law-Rechtskultur erschwert. Der berühmte deutsche Rechtsphilosoph Gustav Radbruch fühn dazu etwas verabsolutierend aus: "Man kann ein englisch-deutsches Rechtswörterbuch nicht aufbauen auf die Obersetzung englischer in entsprechende deutsche Worte, sondern nur auf eine ausführliche Beschreibung der mit den Worten gemeinten Rechtsinstitutionen, so daß aus dem Wörterbuch unversehens ein Rechtslexikon wird."152 Im deutschen rechtsvergleichenden Schrifttum läßt man diese Common Law-Signalwörter deshalb meist unübersetzt, was aufgrund der verwandtschaftlichen Nähe der beiden indoeuropäischen Sprachen und wegen der kulturellen Aufgeschlossenheit gegenüber der englischen Sprache als unbedenklich angesehen wird.

Was den chinesischen Kulturraum anbetrifft, so werden z.B. für das vertypte Delikt (tort) der nuisance 153 sowohl innerhalbHongkongs als auch in der VR China, in Taiwan und in Japan sehr unterschiedliche Schriftzeichenkombinationen als Entsprechungen gewählt. 154 Wenige der zumeist, wie auch im Falle von nuisance, aus zwei Schrift-

149 Z.B.: Beijing Daxue (transl. team), Meiguo Hetongfa, [engl.: "American Contract Law"], Beijing Daxue Chubanshe, Peking 1980; Gao Erlin, Yingmei Hetongfa Gangyao, [engl.: "Essentials of Anglo-American Contract Law"], Nankai Daxue Chubanshe, Tianjin 1984; Pan Qi (transl.), Meiguo Tongyi Shangfadian, [engl.: "The American Uniform Commercial Code"], Zhongguo Duiwai Jingji Maoyi Chubanshe, Peking 1990, Dong Ansheng (et al.), The British Commercial Law (Yingguo Shangfa), Falü Chubanshe, Peking 1991.

150 Z.B.: Andrew Lee / Yang Jen, Outlines of Anglo-American Law of Contracts (Ying Mei Qiyuefa Gailun), Book World Co., Taipeh 1965.

151 Z.B: Tsuyoshi Kinoshita, Ei-Bei keiyakuhou no riron, [engl.: "Theory of Anglo-American Contract Law"], Tokyo Univ. Press, Tokyo 1977.

152 Gustav Radbruch, Der Geist des Englischen Rechts, 4. Auflage, Götingen 1958, S. 8.

153 Nuisance $=$ Belästigung, Beeinträchtigung, Störung (z.B. vom Nachbargrundstück aus durch Rauch, Lärm etc.). Diese und folgende Definitionen nach: C.E. Diell / E. Lorenz, Wörtertuch für Recht, Wirtschaft und Politik, Teil 1: Englisch - Deutsch, 5. Auflage München 1990. Der Geschädigte kann auf Schadensersatz (damages) und ggf. auch auf Unterlassung (injunction) klagen.

154 1) Hongkong: a) Lehrücher: saorao, fangrao; b) Rechtswörtertücher: zirao, fangrao, fang 'ai, fanghai.

2) VR China: fanghai, fanrao(?), sunhai, gonghai, airao, taoyan xing wei. 
zeichen bestehenden Entsprechungen können in dieser Untersuchungsgnuppe als schlichtweg "falsch", weil irreführend angesehen werden. Diese Schriftzeichenkombinationen haben dann bereits Verwendung als chinesische Fachtermini für ein Rechtsinstitut der Civil Law-Rechtsfamilie gefunden, oder sie gehörten bereits im klassischen China zur Rechtssprache. Ansonsten ist die Wahl der "besten" Entsprechung frei und wird demzufolge sehr vom subjektiven Sprachgefühl des Terminologieschöpfers und von der Tiefe seines jeweiligen Rechtsverständnisses geprägt. Nichtchinesen wird hier von einigen Terminologieschöpfem jegliche Kompetenz abgesprochen, Nichtjuristen fühlen sich jedoch durchaus berufen, bei der Terminologieschöpfung mitzumischen.

Besonders von Nichtjuristen wird unter "Mystifizierung" der chinesischen Schriftsprache immer wieder insistiert, daß fast allen Schriftzeichenkombinationen, zumindest aber jedem Schriftzeichen für sich in der chinesischen Kultur ganz allgemein bereits eine mitschwingende Bedeutung innewohne, die die Interpretation zwangsläufig beeinflusse und die von der Bedeutung des westlichen Fachbegriffes grundverschieden sei. Dies wäre aber nur dann relevant, wenn man davon ausginge, daß es den chinesischen Rechtstermini gelingen kann, aus dem langen Schatten ihrer englischen Vorbilder zu reten. Gemäß sec. 10C (1) der Official Languages (Amendment) Ordinance (1987) muß ein chinesischer Terminus gemäß seiner Common Law-Bedeutung ausgelegt werden. Diese Common Law-Bedeutung läßt sich am einfachsten aus der englischen Version und den zugehörigen englischen Präzedenzfällen ermitteln. Die den chinesischen Schriftzeichen gegebenenfalls anhaftende abweichende Bedeutung wird somit per Gesetz für nicht beachtenswert erklärt. Ein Richter, der anders verführe, würde das Recht beugen. Das Gesetz müßte also zunächst geändert werden.

Zum Zeitpunkt des Absterbens der englischen Rechtssprache in Hongkong wird sich die Common Law-Bedeutung der chinesischen Schriftzeichenkombination im chinesischen Kulturkreis jedoch bereits als juristischer Fachterminus mit einer der englischen Version weitgehend entsprechenden Bedeutung durchgesetzt haben, selbst wenn sie die anderen nichtjuristischen Bedeutungen nicht aus der Laiensphäre hat verdrängen können. Diese Wechselbeziehung zwischen Laiensprache und juristischer Fachsprache findet sich übrigens auch in Deutschland. So wird auch der Totschläger als "Mörder" bezeichnet, jede Handlung der Verwaltung ist für den Laien ein "Verwaltungsakt" und vor der an sich überflüssigen Klarstellung in \$ 90a BGB waren zur Empörung der Laienwelt auch Tiere "Sachen", während sie jetzt nur rechtlich wie solche behandelt werden.

Zunächst ist für diese Terminologiegruppe anzuraten, daß eine Harmonisierung der chinesischen Entsprechungen innerhalb Hongkongs erfolgen sollte. Am einfachsten würde dies durch die Erstellung eines offiziellen Rechtsglossares durch die Law Drafting Division. geschehen. Dabei sollte man sich auf eine Ubersetzung pro Fachterminus

3) Taiwan: fanghai, fangrao, sunhai, gonghai, airao, taoyan xingwei.

4) Japan: shenghuo fanghai (Chin. Aussprache der Kan ji), nyuusansu (Katakana-Silhenschrift). 
beschränken. Entgegen der Praxis der existierenden Englisch-Chinesischen Rechtswörterbücher, die eine wenig kreative, unsystematische Anhäufung aller irgendwann einmal aufgetauchten Entsprechungsvarianten vomehmen und dabei vielleicht auch aus kommerziellem Interesse oft einfach nur voneinander abgeschrieben haben, sollte man sich in der Klarheit der Darstellung weitgehend am japanischen Vorbild1 55 orientieren. Die Ergebnisse der Common Law-Forschung in der VR China und auf Taiwan können inhaltlich als Orientierungshilfe dienen, da man in den meisten Fällen wohl aus ihrem unsystematischen Fundus wird auswählen müssen. Die interessante Idee der rein phonetischen Transskription oder der Schaf fung neuer lokaler Schriftzeichen für Common Law-Rechtsinstitute wird sich im politisch-rechtlichen Bereich nicht mit dem Kulturstolz der chinesischen Intellektuellen vereinbaren lassen. Das gleiche gilt für das Unübersetztlassen englischer Fachtermini in sog. "Mischtexten". Es zeigt sich ein Nachteil der ausschließlichen Benutzung der chinesischen Zeichenschrift, der darin besteht, daß China nicht wie Japan auf ein eigens auf ausländische Fremdwörter zugeschnittenes Silbenschriftalphabet (Katakana) zur phonetischen Inkorporation ausländischer Fachtermini zurückgreifen kann.

Uneinheitliche Übersetzung von funktionsähnlichen Rechtsinstituten:

Neben den Common Law-Signalwörtem, deren funktionale Unterschiedlichkeit zum Civil Law eine Sonderbehandlung im Rahmen der Ubersetzungstätigkeit rechtfertigt, gibt es eine große Anzahl von funktionsähmlichen oder -gleichen Rechtsinstituten in den verschiedenen Rechtsordnungen (z.B. contract, offer, acceptance, marriage), die durch ihre länderspezifische verschiedene inhaltliche Ausgestaltung ein wenig voneinander differieren. Diese geringen Abweichungen sind jedoch entweder nicht vorwiegend rechtsfamilienspezifisch oder sic fallen im Vergleich zu den Gemeinsamkeiten nicht so stark als Verwirrungsstifter ins Gewicht, daß sie eine unterschiodliche Úbersetzung zwingend gebieten würden.156 Auch innerhalb einer Rechtsordnung führt die Inhaltsänderung eines Rechtsinstitutes nur selten zu dessen Umbenennung. Es ist unter anderem daran zu erinnem, daß man z.B. das Rechtsinstitut "Ehe" in Deutschland im Laufe der Jahrhunderte nie umbenannt hat, auch wenn sich seine inhaltliche Ausgestaltung veränderte. Die modeme englische Ehe (marriage) kommt der bundesrepublikanischen Ehe wohl inhaltlich näher als die Ehe in der mittelalterlichen Kirchenrechtsordnung. Trotzdem wäre es widersinnig, irgendeiner dieser auf Dauer angelegten Schicksalsgemeinschaften zwischen Mann und Frau den Namen "Ehe" abzusprechen. Was den chinesischen Kulturraum anbetrifft, so finden sich zum Beispiel für das Rechtsinstitut des Vertrages (contract) drei verschiedene chinesische Entsprechungen

155 7.B.: Hideo Tanaka (et al.), Dictionary of Anglo-American Law, Tokyo Univ. Press, Tokyo 1991.

156 Andere Auffassung: E. Epstein, "China and Hong Kong: Law, Ideology and the Future Interaction of the Legal Systems", in: Raymond Warks (ed.), The Future of the Law in Hong Kong, Hongkong 1989 , pp. $61-63$. 
in Hongkong, der VR China, Taiwan, Macao und Japan. ${ }^{157}$ Im Grunde sollte man nach dem oben Gesagten annehmen, daß hier problemlos eine Harmonisierung der Hongkonger Variante mit dem Sprachgebrauch der VR China erfolgen könnte. Bei dem Hongkonger heyue (Vertrag) handelt es sich jedoch um einen symbolträchtigen Schlüsselterminus, an dem sich die Gemüter exhitzen. Hongkong will hier die Besonderheit seines Rechtssystems übertrieben verdeutlichen, um sich symbolisch vor den Festlandjuristen zu schützen, denen vor Augen gefuhrt werden soll, daß im Common Law einfach alles anders ist als gewohnt. Als Argumente für ein Festhalten an diesem Terminus werden die notwendige Berücksichtigung des raditionellen lokalen Rechtssprachgebrauchs in Hongkong, 158 das jüngste kritische Nachdenken bezüglich der Unzulänglichkeit des volksrepublikanischen hetong (Vertrag) in der VR China159 und die Verwechselbarkeit des republikanischen qiyue (Vertrag) mit den Instituten lease (Pacht = zuyue) und deed (gesiegelte Urkunde $=q i j u) 160$ angeführt. Die Law Drafting Division hat sich grundsätzlich, abgesehen von einigen besonderen Landpachtverträgen (sudi qiyue) ${ }^{161}$, für die Beibehaltung des lokalen heyue entschieden, 162 während einige Hongkonger Lehrbuchautoren 163 "in vorauseilendem Gehorsam" auf das volksrepublikanische hetong eingeschwenkt sind.

Abgesehen von diesem interessanten Sonderbeispiel sollte man sich in Hongkong bei dieser Terminologiegruppe (z.B. bei: offer, acceptance) jedoch nur mit guten Gründen gegen eine Ubernahme volksrepublikanischer Termini entscheiden, um den interchinesischen Rechtsverkehr nicht unnötig zu komplizieren.

- Fehlerhafte Ubersetzungen von Common Law-Rechtsinstituten:

Am spektakulärsten ist für den Rechtsvergleicher natürlich das Auffinden von fehlerhaften Ubersetzungen von Common Law-Rechtsinstituten. Nach langem Suchen mit der Lupe empört bzw. erfreut er sich heimlich über die Unkenntnis des Terminologieschöpfers von der unterschiedlichen Funktion ebendieses chinesischen Terminus in der Civil Law-Rechtsf amilie.

157 Hongkong: heyue; VR China und Macao: hetong; Taiwan und Japan: qiyue.

158 Bei Tam Tat Hin, An English and Chinese Dictionary, Hongkong 1875, und bei J. Chalmers, English and Cantonese Dictionary, 7th ed. (H.K.?) 1907, finden sich unter der Rubrik contract allerdings nur die chinesischen Äquivalente hetong, yue und qiyue, (noch?) nicht jedoch heyue.

159 He Weifang, "Qiyue Yu Hetong de Bianxi" [dtsch: "Analyse der Unterschiede zwischen qiyue und hetong"], in: Faxue Yanjiu, Heft 2, 1992, pp. 36-40.

160 Mitunter wird für deed auch einf ach die fehlerhafte Entsprechung qiyue gewählt.

161 Siehe: Interpretation and General Clauses (Chinese Version of Short Titles) Notice, H.K. Govemment Gazette, Legal Suppl. No. 7 vom 24.7.1992, Cap. 150: Xinjie Tudi Qiyue (Xuqi) Tiaoli, [engl.: "New Territories Leases (Extension) Ordinance"]; Cap. 40: Guanqi Tiaoli [engl.: "Crown Leases Ordinance"].

162 Z.B.: Cap. 78 obigen chinesischen Kurztitelverzeichnisses: Wang Xianggang yiwai Diqu Jiuye Heyue Tiaoli, [engl.: "Contracts for Employment Outside Hongkong Ordinance"].

163 Siehe Fn. 148 zu den Vertragsrechtslehrüchem. 
Ohne seine Erkenntnisse empirisch abzusichern ist der Rechtsvergleicher geneigt, von wenigen - mehr oder weniger richtigen - Beispielen ausgehend in unwissenschaftlicher Weise verallgemeinemd meist düstere Prognosen für die Überlebenschancen eines chinesischen Common Law anzustellen. Bei einer tiefergehenden Analyse ganzer Terminologiefelder mag sich jedoch mit großer Wahrscheinlichkeit herausstellen, daß die Zahl der fehlerhaften Übersetzungen gegenüber den durch Funktionsähnlichkeit gerechtf ertigten Ubersetzungen kaum ins Gewicht fällt.

Trotzdem sei hier ausgehend von einem Beispiel von Epstein 164 aus dem Eigentumsrecht eines dieser spektakulären Terminologiefelder näher beleuchtet: So wird der Common Law-Rechtsbegriff personal property fehlerhaft mit dongchan (bewegliches Eigentum) übersetzt, während dongchan im Civil Law der Volksrepublik eher dem deutschen Eigentum an beweglichen Sachen entspricht. Das chinesische "bewegliche Eigentum" ist nicht grundsätzlich, sondem lediglich im Detail abweichend von dem deutschen Eigentum an beweglichen Sachen ausgestaltet, wie z.B. bei den Regelungen über wesentliche Bestandteile und Zubehör. Personal property und das Eigentum an beweglichen Sachen unterscheiden sich jedoch grundsätzlich dadurch, da $\beta$ neben beweglichen Sachen auch Forderungen, Immaterialgüterrechte und das dingliche Pachtrecht (leasehold) in die Kategorie des personal property einzuordnen sind. Noch erschreckender ist die Übersetzung von personal property mit geren caichan, ein Terminus, der in der Volksrepublik bereits als individuelles Eigentum im Gegensatz zu kollektivem und staatlichem Eigentum in Gebrauch ist. Aufgrund oben genannter Rechtsfamilienkonflikte sind auch die chinesischen Übersetzungen anderer Common Law-Rechtsbegriffe in diesem Terminologiefeld wie z.B. real property165, chattels real 166 und chattels personal 167 fehlerhaft. Allgemein gibt es in diesem Terminologiefeld noch keine zufriedenstellenden Übersetzungen. 168 Hier ist eine klare Abrenzung der Hongkonger Termini von denen der Volksrepublik auch auf Kosten der Sprachgewohnheit und Sprachästhetik dringend angezeigt, da es sonst unweigerlich zu "Mißverständnissen" zwischen Hongkonger und festlandchinesischen Juristen kommen wird. Als Arena für solche Terminologiegefechte bei Sachverhalten mit inter-bzw. intrachinesischem Bezug kämen die jeweiligen Gerichte in Betracht, die vor dem Souveräni-

164 Epstein, a.a.O. S. 62 und Fn. 80.

165 Real property = Grundstücksvermögen (freehold). Entspricht nicht dem deutschen Immobiliarsachenrechten, da es aktionenrechtlich entstanden ist (actio in rem) und das dingliche Pachtrecht (leasehold) nicht miturnfaßt.

166 Chattels real $=$ Grundstücksrechte unterhalb von freehold, insbesondere leaschold wegen seiner Ambivalenz als personal property und legal estate.

167 Chattels personal $=$ Umf aßt sämtliches personal property mit Ausnahme von leasehold (bewegliche Sachen, Immaterialgüterrechte, Fordenungen.

168 Zur Not sollte man sich für chinesische "Unwörter" entscheiden. Z.B.: ren zhi caichan (renchan), wu zhi caichan (wuchan), jia de ren zhi caichan (jiarenchan), zhen de ren zhi caichan (zhenrenchan). 
tätswechsel Normen des Intemationalen Privatrechts, danach des Interlokalen Privatrechts anwenden werden. 169

\section{d. Strafrechtsterminologie}

Im Gegensatz zu der schon im klassischen chinesischen Rechtsdenken angeblich vernachlässigten Privarrechtsterminologie, 170 die den "alten Mann in der Sraßenbahn in Causeway Bay"171 weniger interessieren dürfte, hat sich die Hongkonger Strafrechtsterminologie aufgnmd ihrer Wichtigkeit im Alltagsleben bereits mehr gefestigt. In Hongkong haben die chinesischsprachigen Zeitungen172 schon früh Entsprechungen für englische Strafrechtstermini geprägt, die sich im Laufe der Zeit im lokalen Sprachgebrauch eingebürgert haben. Weiterhin kommt der Rechtsquelle der ordinances bei der Benennung von Deliktstatbeständen im lokalen Strafrecht eine zentrale Bedeutung zu, so daß deren Ubersetzungen durch die Chinese Language Division und demnächst durch die Law Drafting Division unter Berücksichtigung des lokalen Sprachgebrauchs einen ordnenden Einfluß ausüben. 173 Leider mangelt es noch an akademischem und kommerziellem Interesse an der Veröffentlichung chinesischsprachiger Lehrbücher über das Hongkonger Strafrecht. 174 Dieses Manko macht sich besonders bei den vorwiegend fallrechtlich definierten allgemeinen Prinzipien des Strafrechts bemerkbar.

Von der Dogmatik und der Methode her gehört das Hongkonger Strafrecht in die Common Law-Rechtsfamilie. Bei den chinesischen Deliktsnamen sind jedoch - wie auch in der Republik China und sogar in der "antif eudalistischen" Volksrepublik, wo lange Zeit jede Vererbbarkeit von Recht vorangegangener Regime negiert wurde - noch Anklänge an den reichen strafrechtlichen Wortschatz früherer chinesischer Dynastien erkennbar. Das gleiche gilt auch für Macao und eingeschränkt für Japan.

169 Siche Volker Pasternak, "Chinese Interlocal Conflict of Laws", LL.M-paper in Civil and Economic Law in the PRC, Faculty of Law, University of Hong Kong 1991.

170 Oft werden die Verträge dabei als Primärquellen unterschätn. Kritisch daz. u:Jörg.Michael Scheil, Chinesische Darlehensverträge aus Turfan-Funden, Göttinger Studien zur Rechtsgeschichte Bd. 20, Göttingen 1992. Vgl. auch: Harro von Senger, Kaufverträge im traditionellen China, Zürich 1970.

171 Beliebte Definition des reasonable man in Hongkonger Juristenkreisen.

172 Historisch: Xunhuan Ribao (Cheungwahn Yahtbou), seit 1874. Aktuell: z.B. Ming Bao (Ming Pao), Dongf ang Ribao (Dungfong Yahtbou), Huaqiao Ribao (Wahkiuh Yahtbou).

173 Hier finden besonders die fast-offiziellen Versionen (s.o.) der Crimes Ordinance (cap. 200), der Offences against the Person Ordinance (cap. 212) und des Organized Crimes Bill (Aug. 1991) Berücksichtigung.

174 Erster Versuch in Hongkong: Li Zong'e, Everyday Law Compendium, a.a.O. vol. 1, cap. 3, pp. 601-782; vgl. VR China: Chu Huaizhi, Meiguo xingfa, [engl.: "American Criminal Law"], Beijing Daxue chubanshe, Peking 1987. 
Weil freiheitliche Prinzipien des westlichen Strafrechts im Denken der Hongkonger zunehmend verwurzelt sind,175 gibt es neben dem Rechtsfamilienkonflikt noch einen sozio-kulturellen, manchmal auch weltanschaulichen Konflikt besonders mit der Volksrepublik China, der durch die Wahl identischer chinesischer Termini eher kaschiert würde. In besonderer Intensität findet sich eine derartige Problematik auch bei der Menschenrechtsterminologie. 176 Bei den Terminologiegruppen kann auf die Ausführungen zur Zivilrechtsterminologie verwiesen werden. Im Strafrecht gibt es jedoch einige Besonderheiten, die im folgenden kurz angeführt seien:

- Uneinheitliche Ubersetzung von Common Law-Signalwörtem:

Als Fremdkörper in der wohl modernsten und aufgeschlossensten chinesischen Gesellschaft Asiens wirken einige archaische Delikte aus viktorianischer Zeit, die weder freiheitlich-demokratischen Ursprungs noch typisch asiatisch sind (z.B.: loitering177), und welche die Phantasie der Terminologieschöpfer besonders angeregt haben. 178

Daneben finden sich im Hongkonger Strafrecht auch einige nicht ganz in diese Gruppe passende "moderne" Delikte oder Delikte im Zusammenhang mit den Aktivitäten der Triaden, deren Begrifflichkeit in der Volksrepublik bisher noch vernachlässigt wird (z.B.: money laundering ${ }^{179}$ ). Hier ist es möglich, daß sich die Hongkonger Termini früher oder später auch in der Volksrepublik durchsetzen werden.

- Uneinheitliche Ubersetzung von funktionsähnlichen Rechtsinstituten:

Ein durchaus anfechtbares Beispiel stellt das Delikt robbery (Raub)180 dar. Es zeigt sich hier aber sehr deutlich, wie sehr die offizielle Hongkonger Version (xingjie)181

175 Siehe Berry Hsu, Fong-chung / Philip W. Baker, Law and Opinion in Hong Kong in the late 1980s, in: Hong Kong Law Journal 1990, No. 3, pp. 345-366. Weitertin: Lau Siu-kai,"The Political Values of the Hong Kong Chinese", in: Peter Wesley-Smith / Albert Chen, The Basic Law and Hong Kong's Future, Hongkong 1988, pp. 19-43.

176 Siehe die Ausführungen dazu im 5. Kapitel.

177 Loitering = Henumlungem bzw. Herumsträunen. Mit Strafe wird bedroht, wer keine überneugende Antwor für sein Verweilen auf öffentlichen Plätzen und in Gebäuden geben kann. Das dann gegen die Grundsätze des in dubio pro reo und der presumption of innocence vermutete kriminelle Ansinnen stellt wohl selbst bei restriktiver Auslegung noch einen Verstoß gegen die Bill of Rights Ordinance dar.

178 Hongkong: youdang (Crimes Ord. sec.160), zujie; VR China: youdang, paihuai, douliu, chiyan; Taiwan: youdang, zujie; Japan: zhiliu; U.S.A.: zai gonggong changsuo xiandang.

179 Money laundering = Geldwäsche. Chin.(nur in Hongkong!): xi heiqian zuixing.

180 Robbery = Raub: Gewaltsame Wegnahme einer fremden beweglichen Sache mit rechtswidriger Zueignungsabsicht. Die verschiedenen raub- und diebstahlsähnlichen Sonderdelikte im klassischen chinesischen Recht, in der VR China und auf Taiwan sollen hier bewußt ausgeklammert bleiben.

181 Organized Crime Bill, schedule 2. Sonstiges (Hongkong): qiang 2 dao, qiang ${ }^{3}$ jie. 
noch in der klassischen chinesischen Rechtsterminologie verwurzelt ist, ${ }^{182}$ während die Volksrepublik hierneue Wege geht (qiang3jie).183

\section{Fehlerhafte Ubersetzungen von Common Law-Rechtsinstituten:}

Fehlerhaft ist zum Beispiel die Hongkonger Ubersetzung von abet 184 (Beihilfe) mit jiaosuo, 185 worunter man in der Volksrepublik Anstiftung versteht.186 Ähnliche Verwimng herrscht bei den Tötungdelikten, wo für den Terminus manslaughter (vorsätzliche Tötung in einem minder schweren Fall) 187 neben dem im klassischen chinesischen Recht mit einer abweichenden Bedeutung belegten wusha (irrtümliche Tötung) 188 auch noch fehlerhafte Ubersetzungen wie guoshi sharen (fahrlässige Tötung) kursieren.

Relevant könnten solche "Mißverständnisse" nach dem Souveränitätsübergang im Interlokalen Strafrecht werden, ggf. auch bei der nicht auszuschließenden Anwendung volksrepublikanischen Staatsschutzstrafrechtes in Hongkong.

\section{Chinesische Menschenrechtsterminologie in Hongkong}

Die chinesische Menschenrechtsterminologie in Hongkong kann aufgrund ihrer politischen Gebundenheit nicht mehr 7.ur chinesischen Common Law-Terminologie im eigentlichen Sinne gezählt werden. Dazu ist sie zu untechnisch und fremdbestimmt. Daran wird wohl auch die in Hongkong erst schwach verwurzelte, ebenfalls fremdbestimmte Terminologie der Bill of Rights Ordinance (1991) nichts mehr ändem können.

182 Das Vokabular des Qing-Codex läßt sich gut anhand der Übersetzung von Guy Boulais, Manuel du Code Chinois, in: Variétés Sinologiques No. 55, Mission Catholique, Shanghai 1924, (kurz: Boulais), erschließen. Weiterhin empfehlenswert: E-tu Zen Sun, Ch'ing Administrative Terms - A Translation of the Terminology of the Six Boards with Explanatory Notes, Harvard University Press, Cambridge 1961, (kurz: Harvard). Hier: Harvard 1860, 1861 (xingjie); BOULAIS: Loi 1061 (qiang2 dao), Comm. suppl. 1092 (qiang2 2 jie).

183 Penal Code, $\S 150$. Taiwan: qiang 2 dao (Penal Code, $\S 328$ ); Japan: qiang2dao (Penal Code, $\S 236)$.

184 Toabet (Verb): Wird meist in der Kombination aiding and abetting verwendet und entspricht dann in groben Zügen der deutschen Beihilfe nebst animus socii, nicht jedoch der Anstiftung.

185 Crimes Ordinance, sec. 38.

186 VR China: Penal Code, § 26; Taiwan: Penal Code, §29; Japan: Penal Code, § 61.

187 Offences against the Person Ordinance, sec. 2.

$188 \mathrm{MJ}$. Meijer, Murder and Adultery in Late Imperial China: A Study of Law and Morality, Sinica Leidensia (vol. 25), Leiden 1991, S. 1, gibt folgende Definition an: wusha = homicide (mousha, dousha) when a bystander had been killed by mistake. Vgl. dazu auch: Erhard Rosner, Die "Zehn schimpflichen Delikte" im chinesischen Recht der Yüan-Zeit, Dissertation München 1964, der wusha auf Fälle der aberratio ictus bezieht. 
Während die offizielle Terminologie im privatrechtlichen und strafrechtlichen Bereich ausschließlich aus lokalen Quellen erschlossen werden kann, entstammt die Hongkonger Menschenrechtsterminologie folgenden verschiedenen Rechtssphären:

1. Bis zum 30.6.1997 und ggf.189 danach: Lokale Bill of Rights Ordinance und dazugehörige Rechtsprechung, subsidiärer Schutz durch Common Law 190.

2. Nach dem 30.6.1997: Grundgesetz und evtl.191 Verfassung der Volksrepublik China.

3. Nach dem 30.6.1997: Gemeinsame Erklärung als völkerrechtlicher Vertrag.

4. Bis zum 30.6.2047: Internationale Menschenrechtspakte (insbes.: Intemational Convenant on Civil and Political Rights). 192

Um nach den blutigen Ereignissen in Peking vom 4.6.1989 die Zuversicht der Hongkonger in die Zukunft wiederherzustellen, gab die Hongkonger Regierung dem Druck der von Bürgerrechtlem, Studenten und Juristen der University of Hong Kong inspirierten lokalen und britischen Medien nach, worauf es schließlich am 8.6.1991 zum Inkraftreten der Bill of Rights Ordinance kam. Durch einen erdrutschartigen Sieg bei den Wahlen zum Legislative Council am 15.9.1991 bewiesen die Bürgerrechtler um Martin Lee, Szeto Wah und Emily Lau, daß sie bei ihrer Kampagne für diese ordinance die Unterstützung breiter Bevölkerungskreise hatten.

Die umstrittene Bill of Rights Ordinance stelt einen unter vielen Vorbehalten stehenden Versuch dar, die International Convenant on Civil and Political Rights, deren völkerrechtliche Weitergeltung durch das Grundgesetz auch für die Zeit nach dem 30.6.1997 garantiert ist, in das lokale Rechtssystem zu transformieren. Dieser Versuch wird von Seiten Pekings heftig kritisiert und durch Drohungen in Frage gestellt, 193 da man befürchtet, daß den durch eine historisch relativierende sozialistische Menschenrechtstheorie auf dem interna-

189 Der Ständige Ausschuß des Nationalen Volkskongresses könnte am 1.7.1997 einen Widerspruch der Bill of Rights Ordinance zum höherrangigen Basic Law gem. Ar. 160 des Grundgesetzes behaupten, und diese daraufhin außer Kraft setzen. Dies widerspräche jedoch dem Geist von Annex 1, Kap. XIII, Abs. 4 der Gemeinsamen Erklärung und von Art. 39 Grundgesetz.

190 Hier in der Bedeutung als Fallrecht (case law).

191 Welche Vorschriften der chin. Veffassung in Hongkong gelten sollen, ist umstritten. Vgl.: Man Depei I Huang Jin, "Zhongguo Quji Falü Chongtu Wenti Yanjiu", [engl.: "Research on Chinese Interlocal Conflict of Laws"], in: Faxue 1989/4, S. 127 Fn. 1; weiterhin: Liu Nanping, "Jiejian Meiguo Zhouxianfa Jiejue Xianggang Jibenfa de Liang Da Nanti zhi Shentao", [engl.: "Deep Discussion on How to Solve the Two Big Difficult Questions of the Basic Law by Analysing the American Federal Constitution"], Faxue Pinglun 1987/4, S. 1-5.

192 Zusammen mit der lokalen Verabschiedung der Bill of Rights Ordinance wurde von britischer Seite auch der Geltungsbereich der Intemational Convenant on Civil and Political Rights auf Hongkong ausgedehnt. Nach dem 30.6.1997 gilt diese zusammen mit der weniger wichtigen International Convenant on Economic, Social and Cultural Rights und den Intemational Labour Conventions kraft Art. 39 des Basic Law für 50 Jahre fort.

193 Siehe: "China Issues Waming on Rights Bill", in: South China Moming Post vom 7.6.1991, p. 1. 
tionalen Parkett verwässerbaren völkerrechtlichen Bestimmungen durch den Hongkonger Transformationsakt plötzlich Schlagkraft zukommen könnte, wenn sie durch die unabhängigen lokalen Gerichte im Sinne einer freiheitlich-universalistischen Menschenrechtstheorie ausgelegt würden. Die chinesische Terminologie der Bill of Rights Ordinance orientiert sich an der bei den Vereinten Nationen registrierten, noch unter dem Einfluß der Republik China auf Taiwan erstellten, offiziellen chinesischen Version der International Convenant on Civil and Political Rights. Man entschied sich in der Law Drafting Division dafür, keine eigene chinesische Menschenrechtsterminologie zu prägen. Ein solcher Schöpfungsakt hätte zwar die Volksrepublik China noch mehr verärgert, wäre aber aus Gründen der Abgrenzung zu dem abzulehnenden Menschenbild der VR China durchaus erwägenswert gewesen.

Die Bürgerrechtsbestimmungen im Grundgesetz entsprechen, von der Terminologie und Konzeption her, denen der Verfassung der Volksrepublik China. Auch die chinesische Version der Gemeinsamen Erklärung orientiert sich terminologisch an deren Bürgerrechtskatalog. Viele Bürgerrechtstermini stammen noch aus republikanischer Zeit. Geändert hat sich dann lediglich deren of fizielle Interpretation, z. B. im Rahmen von Inhaltsbestimmung und Schranken des entsprechenden Bürgerrechtes. Bei den Menschenrechten gibt es deshalb im Grunde keine technisch-terminologischen Mißverständnisse. Die Juristen in der Volksrepublik China und in Hongkong sind sich der unterschiedlichen Interpretation von Menschenrechten in der jeweils anderen Verfassungswirklichkeit sehr bewußt. Dabei betreibt die sorialistische Volksrepublik viel Begrimdungsaufwand für ihre "Menschenrechtstheorie" und versucht so, innen- wie außenpolitisch, aus der argumentativen Defensive in die Offensive überzugehen.

\section{Sprachlicher Stil chinesischer Rechtstexte in Hongkong}

China kann auf eine über 2000 -jährige, reiche literarische Tradition zurückblicken. Die Meisterwerke der chinesischen Klassik und späterer Epochen sind den meisten Chinesen heute nur noch unter Anleitung verständlich. Das gleiche gilt für die klassischen Rechtstexte. Eine gewisse Entfremdung von der literarischen Tradition hat stattgefunden. Grund dafür ist die in der literarischen Revolution im Zusammenhang mit der 4. Mai-Bewegung 194 erfolgte Umorientierung von der klassischen Schriftsprache (wenyan) zur

194 Am 4. Mai 1919 demonstrierten etwa 5000 Studenten in Peking gegen die unbillige Entscheidung der Versailler Friedenskonferenz, das ehem. deutsche Pachtgebiet Kiaochow in der chin. Provinz Shantung der Siegermacht Japan und nicht dem neutralen China zuzuschlagen. Unter dem Begriff "4. Mai-Bewegung" (wusi yundong) wird seither in China jedoch über diesen Anlaß hinaus auch eine neue, mitunter bilderstürmerische Kulturbewegung verstanden (Schlagwort: "Weg mit dem Konfuziusladen!") Thr wohl scharf sinnigster Repräsentant, der "liberale"(Nichukommunist) Hu Shi, (1891-1962) hatte schon 1915 als Student in Comell (U.S.A.) die baihua-Bewegung initiert. Vgl.: 
Umgangssprache (baihwa). ${ }^{195}$ Zur Zeit der 4. Mai-Bewegung war eine solche Veränderung durchaus hilfreich, um die Volksbildung zu steigem und China zu modemisieren. Man konnte im Bereich der Rechtssprache hier sogar an die traditionelle Verwendung von Elementen der baihua in den qingzeitlichen Gerichtsakten anknüpfen. Auf die Spitze getrieben wurde die Sprach-und Schriftreform (1958)196 jedoch in der Volksrepublik China. Auf dem Gebiet der Rechtssprache bildete sich ein umgangssprachlicher, durch marxistische Termini bereicherter Stil heraus, der sich von dem feineren, fachmännischen Stil der republikanischen Periode abgrenzen läBt. Seit Einleitung der Dengschen Modemisierungspolitik (1978) finden sich besonders in den Entscheidungen des Obersten Volksgerichtshofes allerdings wieder vereinzelt Elemente des wenyan. 197 Den republikanischen Stil, der mitunter auch als Briefstil, xinwen $t i 198$ oder gehobener Stil bezeichnet wird, kann man in viel ausgeprägterer Form noch in der Rechtssprache Hongkongs, Taiwans und Macaos finden.

Die meisten Intellektuellen in Hongkong, die die kantonesische Sprache schon immer als wahre Hüterin der sprachlichen Traditionen Chinas verstanden haben, 199 stehen der "primitiven", politisierten Rechtssprache der Volksrepublik ablehnend gegenüber. Um dem Recht den ihm gebührenden Status und Respekt in einer hierarchischen chinesischen Gesellschaft zukommen zu lassen, müsse die Rechtssprache in einem zwar verständlichen, aber gehobenen Schriftchinesisch abgefaßt sein. Was unter gehobener Schriftsprache zu verstehen ist, wird jedoch durchaus unterschiedlich beurteilt. Allgemein hingenommen wurde, daB bei einer Änderung der Richtlinien für den Dokumentar-und Briefstil vom März 1983 einige archaische Ausdrücke des wenyan in der of fiziellen Kommunikation abge-

Imnnamuel C.Y. Hsü, The Rise of Modem China, Oxford Univ. Press, 3rd. ed. Oxford/New York 1983, pp. 493-513.

195 Ein halb-klassischer, halb-umgangssprachlicher "joumalistischer Stil" (xinwen ti) findet sich schon seit der "Hunder Tage-Reform" im Jahre 1898 bei dem qingzeitlichen Reformer Liang Qichao (1873-1929); vgl.: Hsü, a.a.O. pp. 501, 425.

196 In der Volksrepublik wird eine einf acher zu erlemende "proletarische" baihua propagiert. Die vereinfachten Schriftzeichen (jiantizi) sind leider seinerzeit nicht mit den japanischen Kurzvarianten der Kanji harmonisien worden; vgl.: Wolfram Eberhard, Geschichte Chinas: von den Anfägen bis zur Gegenwart, Kröner Verlag, 3. Auflage Stuttgart 1980, S. 378-381.

197 Siehe: Erhard Rosner, Schriftsprache: Studien zur Diglossie des modemen Chinesisch, Chinathemen Band 74, Bochum 1992, S. 127-130.

198 Siehe Fn. 195.

199 Das Kantonesische habe die Aussprache des Chinesischen früherer Dynastien (Tang) konserviert; siehe: $S \boldsymbol{R}$. Ramsey, The Languages of China, Princeton 1987, S. 98-99. Indiz für diese These stellt die Tatsache dar, daß sich Gedichte der glanzvollen Tang-Dynastie (618-907 n. Chr.) mitunter nur noch im Kantonesischen reimen. Die kantonesische Bezeichnung für die chinatowns in fremden Staaten ist demnach auch "Straße der Tang-Menschen" (Tohngyahn gaai = Tangren jie), während die Nordchinesen die chinesische Sprache als "Han-Sprache" (Hanyu) bezeichnen (Han-Dynastie: 206 v.Chr -221 n. Chr.). 
schafft wurden. ${ }^{200}$ Dies sollte man aber eher als Maßnahme für mehr Transparenz und Volksnähe der Verwaltung, denn als bewußte Angleichung an den Stil der Volksrepublik in vorauseilendem Gehorsam betrachten.

Die ordinances, die durch die Law Drafting Division erstellt werden, sind die wichtigstige schriftliche chinesischsprachige Rechtsquelle in Hongkong. Im Stil ähneln sie der in Taiwan verbreiteten Rechtsprache. Dabei wird unter Verwendung von Langzeichen eine kompaktere Ausdrucksweise (z.B.: dan, ru; statt: danshi, ruguo) nebst Verwendung schriftsprachlicher Partikel (wie z.B.: ji, yu, yi, er) bevorzugt. Während die Chinese Language Division noch eisern an der klassischen Schlüsselpartikel zhi zur Bezeichnung der "Genitivkonstruktion" festgehalten hatte, hat sich die Law Drafting Division allerdings für einen etwas modemeren "Mischstil" unter Verwendung der umgangssprachlicheren Partikel de entschieden.

Bei der Chinese Digest, wie auch im Gabinete para a traduçao jurídica in Macao bestehen die volksrepublikanischen Berater auf "Hamonisierung" des Stils der lokalen Rechtssprache mit dem des zukünftigen Souveräns, der Volksrepublik China. Hongkong sei ein Teil Chinas und die chinesische Bevölkerung müsse das Hongkonger Recht ohne Mühe verstehen können. Die lokalen Anhänger eines gehobeneren Stils und Berater aus Taiwan argumentieren, daB die chinesische Sprache in proletarischer Hand verkommen sei und früher oder später auch in der Volksrepublik einem kultivierteren Stil weichen werde. Häufig wird auch kritisiert, daß man durch die Verwendung von Kurzzeichen noch mehr von der kulturellen Tradition entfremdet werde. Außendem sei es unter der Formel "ein Land, zwei Systeme" völlig irrelevant, ob das chinesische Volk das Recht Hongkongs verstehe. Entscheidend käme es auf die Bedürfnisse der kantonesischen Bevölkerung Hongkongs an. In der Volksrepublik interessierten sich ohnedies nur Experten und Politiker für das Hongkonger Recht. Denen müsse aber eine Lektüre des Hongkonger Rechtsstils nebst Anblick von Langzeichen zuzumuten sein. Persönliche Eitelkeiten und Ambitionen auf beiden Seiten spielen hier sicherlich ebenfalls eine gewisse Rolle, wenngleich die Hongkonger Argumente die besseren zu sein scheinen.

Die baihua-Bewegung nach dem Sturz des Kaiserreiches hat in Hongkong nie auf uneingeschränkte Sympathie stoßen können, da mit baihua die Umgangssprache Pekings oder Nankings gemeint ist, nicht jedoch die Umgangssprache Kantons oder Hongkongs. Während das wen yan für alle gebildeten Chinesen gleichermaßen ein zu erlernender Kunststil war, ist für die Hongkonger durch die baihua die Frage aufgeworfen worden, warum man denn nun in der fremden Pekinger oder Nankinger Umgangssprache schreiben lemen solle. Zwar war eine feinere Form dieser fremden Umgangssprache in den besseren Kreisen

200 Vgl.: Chen Yaonan, Yingyongwen Gaishuo [dtsch.: "Überblick über die Gebrauchsprosa"], Hong Kong 1991, Anhang; (Zitien bei: Erhard Rosner, a.a.O. S. 131). 
schon zuvor als Beamtensprache (guanhua) ${ }^{201}$ in Südchina in Gebrauch, deren Bedeutung als Standard trat jedoch hinter der schriftlichen, optischen Kommunikation zurück.202 Letztlich führte somit erst die Aufwertung der baihua dazu, das Kantonesische im Vergleich zum Mandarin of fiziell herabzustufen. Nicht realisierbar erscheint die wohl sezessionistische Idee, eine kantonesische baihua (written Cantonese203) unter Verwendung von lokalen Schriftzeichen salonfähig zu machen.

\section{Ausblick}

Welche Bedeutung schon im klassischen chinesischen Denken der exakten Terminologiewahl und nötigenfalls der "Richtigstellung der Begriffe"204 zukam, wird aus folgender Textstelle205 deutlich:

Dsi Lu sprach: "Der Fürst von We wartet auf den Meister, um die Regierung auszuüben. Was würde der Meister zuerst in Angriff nehmen?"

Der Meister sprach: "Sicherlich die Richtigstellung der Begriffe."

Dsi Lu sprach: "Darum sollte es sich handeln? Da hat der Meister weit gefehlt! Warum denn deren Richtigstellung?"

Der Meister sprach: "Wie roh du bist, Yu! Der Edle läßt das, was er nicht versteht, sozusagen beiseite. Wenn die Begriffe nicht richtig sind, so stimmen die Worte nicht;206 stimmen die Worte nicht, so kommen die Werke nicht zustande; kommen die Werke nicht zustande, so gedeiht Moral und Kunst nicht; gedeiht Moral und Kunst nicht, so treffen die Strafen nicht; treffen die Strafen nicht, so weiß das Volk nicht, wohin Hand und Fuß setzen. ..."

Machte man sich ein solches Zitat nun - in Manier eines chinesischen Gelehrten mit klassischer Bildung - für die aktuelle sprachpolitische Kontroverse zu Nutze, so könnte man es als Frage verkleidet - etwa wie folgt auf Hongkong beziehen: Wer stellt wann im englisch-

201 Davon ist der in Hongkong verbreitete englische Teminus Mandarin abgeleitet, der heute im Grunde nur noch eine altmodische Bezeichnung für Putonghua bzw. Guoyu darstellt.

202 Siehe: Erhard Rosner, a.a.O. S. 2.

203 Vgl.: R.S. Bauer, a.a.O. S. 245-293.

204 Chin.: theng ming. Im Deutschen genauer mit Ad justierung der Namen oder mit Rektifikation der Bezeichnungen übersetzbar. Theng ming stellt einen Schlüsselbegriff konfuzianischen Denkens in einer Zeit des gesellschaftlichen Umbruchs und des Verfalls der Sitten dar.

205 Konfuzius (551-479 v. Chr.) in den Gesprächen (Lun Yü); Buch XII, 3 (Dsi L.u); übersetzt von Richard Wilhelm, Eugen Diederichs Verlag, Jena 1923, S. 135.

206 "Ming bu zheng, ze yan bu shun;"- Der berühmte schottische Sinologe James Legge (1814-1897) übersetzt diese Textstelle mit: "If names be not correct, language is not in accordance with the truth of things;"- siehe: The Chinese Classics, in 5 vol., vol. 1: Confucian Analects, The Great Leaming and the Dactrine of the Mean, Hongkong 1861, 2nd. ed. Oxford (1893-95), repr. Hong Kong Univ. Press 1961. 
sprachigen Hongkonger Rechtssystem die Begriffe in chinesischer Sprache richtig, damit die chinesische Bevölkerung weiß, wohin Hand und Fuß setzen?

Erst spät hat sich die Hongkonger Regierung emsthaft um ein Common Law in chinesischer Sprache bemüht. Die Anstrengungen stehen in direktem Zusammenhang mit der politischen Interessenartikulierung der wachsenden Mittelschicht207 Hongkongs in Anbetracht des nahenden Souveränitätswechsels. Mißtrauen gegenüber den neuen Herren aus Peking veranlaßte die Hongkonger Regierung und einige namhafte Juristen, die chinesischen Termini noch kurz vor dem Machtwechsel selber zu prägen, damit die Begriffe des Common Law nicht nach dem 30.6.1997 vom nördlichen Nachbam richtiggestellt würden.

Die einzigartige Situation des Chinesischen als Rechtssprache in Hongkong bietet dem vergleichenden Rechtswissenschaftler und Sinologen günstiges Anschauungsmaterial für allgemeinere Aussagen über den Zusammenhang zwischen Sprache und Recht. Vielleicht kann eine solche vergleichende Rechtslinguistik sogar einen Anstoß für Neurologen, Informatiker und Psychologen geben, indem sie die richtigen Fragen über das menschliche Denken stellt. Weithin ungeklärt ist nämlich, wie das menschliche Denken genau funktioniert. Inwieweit wird die Information, nachdem sie durch audio-visuelle Kanäle ins Gehim gelangt ist, vom Kommunikationsmedium Sprache abgetrennt und als Konzept oder Struktur gespeichert? Inwieweit ist eine solche sprachballastfreie Information durch die Benutzung einer anderen Sprache als Kommunikationsmedium mitteilbar? Welchen Einfluß haben die "primitive" Grammatik208 und die ideographische Schrift der Chinesen auf das Denken?209 Welchen Einfluß hat die unterschiedliche Beschaffenheit und Anzahl kulturell erlemter Grundinformationen im menschlichen Gehim auf die Speicherung und Strukturierung neuer, unbekannter Information? Differieren die Individuen eines Volkes vielleicht sogar mehr als die Völker im Durchschnitt untereinander? Spielen himphysiologische Erbfaktoren eine Rolle?

207 So ist z.B. das durchschnittliche Monatseinkommen eines Haushaltes von HK\$ 2955 im Jahre 1981 (1,24 Mio. Haushalte) über HK\$ 5160 im Jahre 1986 (1,45 Mio. Haushalte) auf HK\$ 9964 (1,58 Mio. Haushalte) angewachsen. 1991 verf ügten bereits $28,9 \%$ der Haushalte über mehr als HK\$ 15000 im Monat (vgl. 1980: 2,3\%). Inflationsrate ca. $10 \%$; HK\$ 5 ensprechen ca. 1 DM. Bei niedrigen Steuem und Lebenshaltungskosten sind z.T. hohe Wohnkosten anzusetzen (am härtesten treffen diese die sog. "sandwich class", die nicht mehr in den Genuß von Sozalwohnungen kommt). Siehe: Hong Kong 1991 Population Census (Fn. 5), Table 25, p. 60.

208 Kontrovers: Alfred Bloom, The Linguistic Shaping of Thought: A Study in the Impact of Language on Thinking in China and the West, Hillsdale (N.J.) 1981.

209 Die These vom visuellen Denken der Chinesen wird durch ihr im Vergleich zu Amerikanem besseres Abschneiden in visuellen Intelligenztests (z.B. Raven's Progressive Matrices) untermauert. So jedenf alls: Michael H. Bond, Beyond the Chinese Face - Insights from Psychology, Hongkong 1991, pp. 27-28. 
All diese offenen Fragen sind für die Übersetzung von Literatur weniger entscheidend, soweit das ästhetische Moment im Vordergrund steht. Auf dem Gebiet der Rechtssprache, besonders bei einem so listigen Kulturvolk wie den Chinesen, 210 könnten jedoch selbst kleine Bedeutungsnuancen von großer Konsequenz für die Zukunft Hongkongs sein. Falls, wie hier unterstellt, eine bedeutungsverlustlose Úbersetzung von Rechtskonzepten überhaupt theoretisch möglich ist, wird die Geschichte zeigen, ob die Hongkonger Anstrengungen zur Verhinderung einer bedeutungsverschiebenden Sinisierung des Common Law ausreichend und noch rechtzeitig waren, um das Common Law in den Köpfen der Hongkonger fest zu verankem. Nachdenklich stimmt der Präzedenzfall der bedeutungsverschiebenden Sinisierung des indischen Buddhismus in China.211 Skeptisch stimmt, daß die kantonesische Muttersprache als schriftliche Rechtssprache vernachlässigt wird. Ob ein Absterben des Common Law allerdings unbedingt schlecht für Hongkongs Zukunft sein würde, hinge auch davon ab, welchen Stimulus es dem $t i$ (der Essenz)212 der chinesischen Kultur vor seinem Tode noch hat geben können.

210 Empfehlenswerte Lektüre zu diesem Thema: Harro von Senger, Strategeme, Lebens- und Überlebenslisten der Chinesen - die berühmten 36 Strategeme aus drei Jahnausenden, Schern. Verlag, 8. Aufl. Bem/München/Wien 1992.

211 Vgl.: W. Bauer, China und die Hoffnung auf Glück. Paradiese, Utopien, Idealvorstellungen, München 1971 (Tb.:74), S. 216 ff. Weiterhin: Eric Zürcher, The Buddhist Conquest of China, the Spread and Adaption of Buddhism in Early Medieval China, 2 Bde, Leiden 1959.

212 Die von Deng Xiaoping revitalisiene Theorie des Zhang Zhidong (1837-1909) bezüglich einer selektiven Modemisienung durch selektives Lemen vom Westen (thongxue wei ti, xixue wei yong) [dtsch.: "Das chinesische Wissen als Grundlage, das westliche Wissen für praktische Zwecke"] scheint auf dem Gebiet des Rechts nicht zu greifen, da die Rezeption fremden Rechts kein rein technischer Prozeß ist, sondem auf lange Sicht auch die Essenz chinesischer Kultur beeinflußt. Vgl. in diesem Zusammenhang: Erhard Rosner, China und der Westen: Historische Perspektiven eines aktuellen Problems, Göttingen 1982, S. 12-20. 
Entsprechungen im chinesischen Sprachraum für ausgewählte englische Common Law-Termini

\section{1. nuisance}

\begin{tabular}{|c|c|c|c|c|}
\hline $\begin{array}{l}\text { 䢂摄 } \\
\text { saorao }\end{array}$ & $\begin{array}{l}\text { 湏摄 } \\
\text { fanrao }\end{array}$ & $\begin{array}{l}\text { 妨野 } \\
\text { fangai }\end{array}$ & $\begin{array}{l}\text { 妨按 } \\
\text { fangrao }\end{array}$ & $\begin{array}{l}\text { 损害 } \\
\text { sunhai }\end{array}$ \\
\hline $\begin{array}{l}\text { 妨害 } \\
\text { fanghai }\end{array}$ & 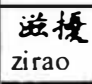 & $\begin{array}{l}\text { 公害 } \\
\text { gonghai }\end{array}$ & 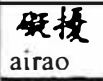 & 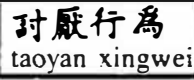 \\
\hline
\end{tabular}

2. contract

\begin{tabular}{|l|l|l|}
\hline $\begin{array}{l}\text { 合约 } \\
\text { heyue }\end{array}$ & $\begin{array}{l}\text { 合同 } \\
\text { hetong }\end{array}$ & $\begin{array}{l}\text { 梁約 } \\
\text { qiyue }\end{array}$ \\
\hline
\end{tabular}

3. personal property

\begin{tabular}{|c|c|c|}
\hline $\begin{array}{l}\text { 勒童 } \\
\text { dongchan }\end{array}$ & $\begin{array}{l}\text { 㑭人财㞗 } \\
\text { geren caichan }\end{array}$ & $\begin{array}{l}\text { 人之財産 } \\
\text { ren zhi caichan (renchan) }\end{array}$ \\
\hline
\end{tabular}

\section{4. loitering}

\begin{tabular}{|c|c|c|}
\hline $\begin{array}{l}\text { 游蓄 } \\
\text { youdang }\end{array}$ & $\begin{array}{l}\text { 逶留 } \\
\text { douliu }\end{array}$ & $\begin{array}{l}\text { 滞留 } \\
\text { zhiliu }\end{array}$ \\
\hline $\begin{array}{l}\text { 阻椾 } \\
\text { zujie }\end{array}$ & $\begin{array}{l}\text { 遇延 } \\
\text { chiyan }\end{array}$ & $\begin{array}{l}\text { 在公共場所闌药 } \\
\text { zai gonggong changsuo } \\
\text { xiandang }\end{array}$ \\
\hline
\end{tabular}

\section{5. robberv}

\begin{tabular}{|c|c|c|c|}
\hline $\begin{array}{l}\text { 行劫 } \\
\text { xingjie }\end{array}$ & $\begin{array}{l}\text { 强盗 } \\
\text { giang }{ }^{2} \mathrm{dao}\end{array}$ & $\begin{array}{l}\text { 棯动 } \\
\text { giang } 3 \text { jie }\end{array}$ & $\begin{array}{l}\text { 唅盗 } \\
\text { qiang }{ }^{3} \text { dao }\end{array}$ \\
\hline
\end{tabular}

\section{6. abet}

\begin{tabular}{|l|l|l|}
\hline $\begin{array}{l}\text { 教筑 } \\
\text { jiaosuo }\end{array}$ & $\begin{array}{l}\text { 支持 } \\
\text { zhichi }\end{array}$ & $\begin{array}{l}\text { 接助 } \\
\text { ywanzhu }\end{array}$ \\
\hline
\end{tabular}

\section{7. manslaughter}

\begin{tabular}{|c|c|c|}
\hline $\begin{array}{l}\text { 談般 } \\
\text { ushat }\end{array}$ & 過失投人 & $\begin{array}{l}\text { 般人 } \\
\text { sharen }\end{array}$ \\
\hline
\end{tabular}


Chinese as the Language of the Law in a Common Law Context: The Case of Hong Kong

\section{By Volker Pasternak}

The British Crown Colony of Hong Kong is preparing for the Chinese takeover on July 1 , 1997, when it will become a Special Administrative Region under Art. 31 of the constitution of the People's Republic of China (PRC). The law of Hong Kong belongs to the Common Law family. The official language of the law used to be exclusively English. Since April 4, 1987 the Hong Kong govemment and some legal scholars are speeding up the process of establishing a bilingual legal system in Hong Kong. Both the Sino-British Joint Declaration and the Basic Law provide for the maintenance of the Common Law in Hong Kong until June 30, 2047. "Chinese" (which could mean Mandarin and/or Cantonese) will be the future language of the law, whereas the future status of the English language remains unclear.

This article is a first attempt to contribute to the current debate of the relationship between language and law, thereby trying to learn more about the cultural conditionality of human thinking. To this end, Chinese Common Law terminology and the style of written "Law Chinese" in Hong Kong will be analysed.

The article will look back at the historic roots of Chinese Common Law terminology, and then analyse the current legal framework and the future prospects of a bilingual legal system in Hong Kong. Later, the article will focus on a comparison of Chinese legal terminology in Hong Kong, the PRC, Taiwan, Macao and Japan in the following three branches of the law: Private Law, Criminal Law and Human Rights Law. On this basis of the backgrounds and objectives of the different players in the Hong Kong legal terminology game, some possible solutions to terminology conflicts arising from different legal families or political systems will be put forward. Mainland Chinese criticism of the style of written "Law Chinese" in Hong Kong is placed into a broader context and the impact of neglecting the local Cantonese language in the written law is also considered.

Although the Common Law might finally die out in Hong Kong, it will be a source of inspiration for a future Chinese legal culture. 